\title{
Galvanostatic Intermittent Titration and Performance Based Analysis of $\mathrm{LiNi}_{0.5} \mathrm{Co}_{0.2} \mathrm{Mn}_{0.3} \mathrm{O}_{2}$ Cathode
}

\author{
Ankit Verma, ${ }^{\mathrm{a}, *}$ Kandler Smith, ${ }^{\mathrm{b}, * *, \mathrm{z}}$ Shriram Santhanagopalan, ${ }^{\mathrm{b}}$ Daniel Abraham, ${ }^{\mathrm{c}, * *}$ \\ Koffi Pierre Yao, ${ }^{\text {c,***}}$ and Partha P. Mukherjee ${ }^{\mathrm{a}, * *, \mathrm{z}}$
}

\author{
${ }^{a}$ School of Mechanical Engineering, Purdue University, West Lafayette, Indiana 47907, USA \\ ${ }^{b}$ National Renewable Energy Laboratory, Golden, Colorado 80401, USA \\ ${ }^{c}$ Argonne National Laboratory, Argonne, Illinois 60439, USA
}

\begin{abstract}
Galvanostatic intermittent titration technique (GITT) - a popular method for characterizing kinetic and transport properties of battery electrodes - is predicated on the proper evaluation of electrode active area. $\mathrm{LiNi}_{0.5044} \mathrm{Co}_{0.1986} \mathrm{Mn}_{0.2970} \mathrm{O}_{2}$ (NCM523) material exhibits a complex morphology in which sub-micron primary particles aggregate to form secondary particle agglomerates. This work proposes a new active area formulation for primary/secondary particle agglomerate materials to better mimic the morphology of NCM532 electrodes. This formulation is then coupled with macro-homogeneous models to simulate GITT and half-cell performance of NCM523 electrodes. Subsequently, the model results are compared against the experimental results to refine the area formulation. A single parameter, the surface roughness factor, is proposed to mimic the change in interfacial area, diffusivity and exchange current density simultaneously and detailed modeling results are presented to provide valuable insights into the efficacy of the formulation. (C) The Author(s) 2017. Published by ECS. This is an open access article distributed under the terms of the Creative Commons Attribution Non-Commercial No Derivatives 4.0 License (CC BY-NC-ND, http://creativecommons.org/licenses/by-nc-nd/4.0/), which permits non-commercial reuse, distribution, and reproduction in any medium, provided the original work is not changed in any way and is properly cited. For permission for commercial reuse, please email: oa@electrochem.org. [DOI: 10.1149/2.1701713jes] All rights reserved.

(c)) BY-NC-ND
\end{abstract}

Manuscript submitted July 31, 2017; revised manuscript received October 16, 2017. Published November 3, 2017.

Lithium ion batteries (LIBs) are ubiquitous in energy storage applications. LIBs are versatile and have completely penetrated the consumer electronics market involving low power applications, e.g. mobile phones and laptops. ${ }^{1}$ In recent years, the use of LIBs in high power applications like electric vehicles is showing great promise. ${ }^{2}$ Consequently, vigorous efforts are being directed toward improving its capacity and rate capabilities. LIB energy and power density is directly related to the constituent anode/cathode chemistries. The couples are chosen such that potential difference between the electrodes and $\mathrm{Li}^{+}$ ions storage capacity are maximized. Additionally, fast intercalation and diffusion in the solid phase are required for high energy efficiency at high power demands. For anode, graphite has proved to be a valuable material with maximum theoretical capacity estimated at 372 $\mathrm{mAh} / \mathrm{g}_{\text {graphite }}$ combined with open circuit potential close to $0.0 \mathrm{~V}$ vs $\mathrm{Li}$ for wide range of state of charge. Graphite as an anode material shows robust cycling performance, decent rate capabilities and satisfactory thermal stability. ${ }^{3,4}$ Current cathodes generally exhibit lower theoretical capacity compared to anodes. Thus, a significant share of research efforts have been concentrated on finding and characterizing novel LIB cathode materials.

Several materials have been scrutinized in the past to function as the cathode in LIB. Transition metal oxides such as $\mathrm{LiCoO}_{2}{ }^{5}$ $\mathrm{LiMn}_{2} \mathrm{O}_{4},{ }^{6} \mathrm{LiFePO}_{4},{ }^{7} \mathrm{LiNi}_{0.8} \mathrm{Co}_{0.2} \mathrm{O}_{2},{ }^{8} \mathrm{Li}_{1-\mathrm{x}}\left(\mathrm{Ni}_{1-\mathrm{y}-\mathrm{z}} \mathrm{Co}_{\mathrm{y}} \mathrm{Mn}_{\mathrm{z}}\right) \mathrm{O}_{2}(\mathrm{M}$ $=\mathrm{Al}, \mathrm{Mg}),{ }^{9} \mathrm{Li}_{3} \mathrm{~V}_{2}\left(\mathrm{PO}_{4}\right)_{3}{ }^{10}$ etc. form the majority of the materials researched. Lately, the Ni-Mn-Co (NMC) family of oxides is generating significant interest owing to its good thermal stability and rate characteristics. ${ }^{11}$ Several variants of the NMC family have emerged over the past decade some of which are listed here: $\mathrm{Li}\left(\mathrm{Ni}_{0.33} \mathrm{Mn}_{0.33} \mathrm{Co}_{0.33}\right) \mathrm{O}_{2},{ }^{12,13} \mathrm{Li}\left(\mathrm{Ni}_{0.6} \mathrm{Mn}_{0.2} \mathrm{Co}_{0.2}\right) \mathrm{O}_{2},{ }^{14,15}$ $\mathrm{Li}\left(\mathrm{Ni}_{0.8} \mathrm{Mn}_{0.1} \mathrm{Co}_{0.1}\right) \mathrm{O}_{2}, \mathrm{Li}\left(\mathrm{Ni}_{0.5} \mathrm{Mn}_{0.3} \mathrm{Co}_{0.2}\right) \mathrm{O}_{2}{ }^{16-18}$ etc.

Amongst the aforementioned cathode materials in the NMC family, $\mathrm{Li}\left(\mathrm{Ni}_{0.33} \mathrm{Mn}_{0.33} \mathrm{Co}_{0.33}\right) \mathrm{O}_{2}{ }^{19}$ and $\mathrm{Li}\left(\mathrm{Ni}_{0.5} \mathrm{Mn}_{0.3} \mathrm{Co}_{0.2}\right) \mathrm{O}_{2}{ }^{20}$ have already been adopted for commercial use. However, there is a dearth of literature focused on characterization of $\mathrm{Li}\left(\mathrm{Ni}_{0.5} \mathrm{Mn}_{0.3} \mathrm{Co}_{0.2}\right) \mathrm{O}_{2}$ (NCM523) cathode material as compared to $\mathrm{Li}\left(\mathrm{Ni}_{0.33} \mathrm{Mn}_{0.33} \mathrm{Co}_{0.33}\right) \mathrm{O}_{2}(\mathrm{NMC} 333)$ cathodes. NMC333 has been the focus of several articles related to synthesis, kinetic and transport properties estimation and performance rate study. ${ }^{13,21}$ For NCM523, a few characterization studies have come out in the past decade. Yang et al. ${ }^{22}$ used Galvanostatic Intermittent

\footnotetext{
*Electrochemical Society Student Member.

**Electrochemical Society Member.

${ }^{\mathrm{z} E}$-mail: kandler.smith@nrel.gov; pmukherjee@purdue.edu
}

Titration Technique (GITT) and Electrochemical Impedance Spectroscopy (EIS) to determine the diffusion coefficient of $\mathrm{Li}$ ions in spherical NCM523. Both these methods require the accurate quantification of electroactive area for subsequent computation of diffusivity. The authors reported three different computations for Li diffusion coefficient values based on cell geometric area, BET surface area and electrochemical active area (assumed as half of BET area). Consequently, there is three orders of magnitude variation in the reported values. Similar diffusivity calculations as well as charge transfer resistance values for $\mathrm{Li}_{1.12}\left[\mathrm{Ni}_{0.5} \mathrm{Co}_{0.2} \mathrm{Mn}_{0.3}\right]_{0.89} \mathrm{O}_{2}$ were reported by Bai et al., ${ }^{23}$ using GITT and EIS. Here again, the geometric surface area of the electrode is used for diffusivity computations which is fallacious. Although the above results give reasonable macro trends for diffusivity variation with state of charge, the diffusivity magnitudes have wide variations depending on the active area definition used. Further diffusivity computations for NCM523 have been undertaken by Kong et al. ${ }^{24}$ (EIS), Xia et al. ${ }^{25}$ (EIS) and Amin et al. ${ }^{26}$ (EIS and GITT at multiple temperatures).

It is apparent that GITT and EIS have emerged as robust electrochemical techniques for battery material characterization. However, extraction of accurate kinetic and transport quantities from GITT and EIS necessitates the precise computation of interfacial area, which gets even more complicated for NCM523 particle agglomerates exhibiting bimodal particle size distribution. Thus, it becomes imperative to design first an accurate mathematical descriptor of active area for NCM523 electrodes. This estimate is then coupled with macro homogeneous performance models to simulate GITT and half-cell performance of NCM523 electrodes. Refinement of the area estimates is executed subsequently to obtain best fit of the simulated performance curves with experimental datasets. The beauty of the method lies in the seamless coupling between GITT and half-cell performance experiments and simulations using scientific descriptors for active area.

In this work, we have synergized GITT and half-cell performance experiments with simulations utilizing macro homogeneous models and active area predictors. The objective of this work is to extract accurate kinetic and transport properties from experimental GITT datasets which can be further used in half-cell performance studies. To the best of our knowledge, this work is the first modelling study on performance of NCM523 electrodes. The model serves two purposes; firstly, it refines battery material property data extraction from experimental GITT/half-cell performance datasets; and secondly, it can be used as a predictive tool to estimate NCM523 battery performance under different operating conditions. 


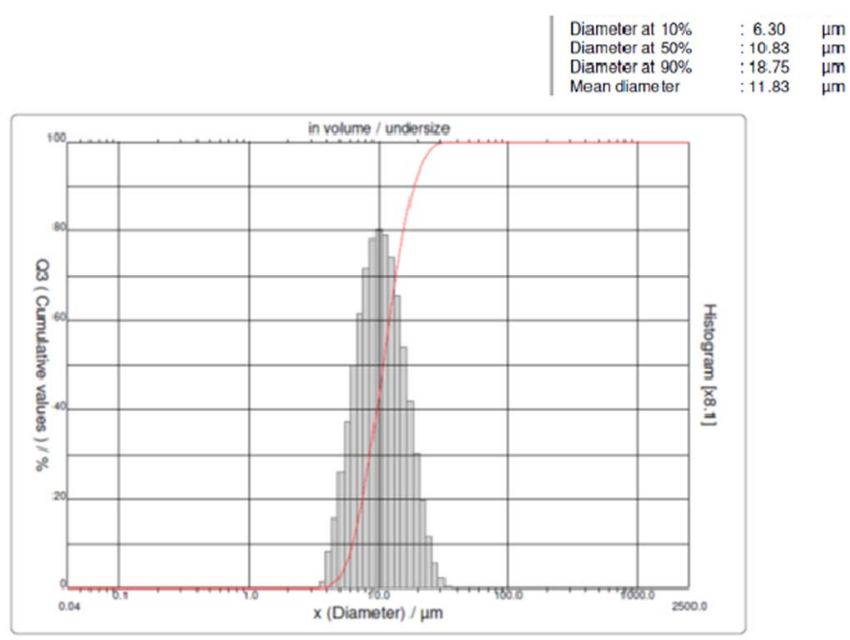

Figure 1. Particle Size Distribution of TODA NCM523 powder.

The rest of the article is divided as follows. Details of the experimental method are elaborated first. Analysis of the experimental GITT datasets using area independent formulations are performed to obtain the state of charge dependence of chemical diffusion coefficient and exchange current density for NCM523 material. Subsequently, active area formulation for bimodal NCM523 agglomerate particles is elucidated. The area formulation is then utilized in tandem with state of charge dependent diffusivity and $\mathrm{Li}$ intercalation rate constant inside macro homogeneous single particle model for obtaining match with GITT experimental datasets. Afterwards, both single particle model and pseudo $2 \mathrm{D}$ model is used in conjunction with the property relations to corroborate the experimental NCM523 half-cell constant current discharge performance.

\section{Experimental}

The NCM523 oxide material was obtained from TODA America. The particle size distribution (PSD) of the material is given in Figure 1. NCM523 powder exhibits spherical secondary particle morphology with dimensions in the range of 6 to 20 microns. The primary particles are of the order of 0.2 to 2 microns. The BET area of the powder is $0.28 \mathrm{~m}^{2} / \mathrm{g}^{27}$

Galvanostatic Intermittent Titration Technique (GITT) and halfcell performance studies were performed at the Cell Analysis, Modeling and Prototyping (CAMP) facility of Argonne National Lab (ANL) with MACCOR cyclers on $\mathrm{Li} \mid \mathrm{LiNi}_{0.5044} \mathrm{Co}_{0.1986} \mathrm{Mn}_{0.2970} \mathrm{O}_{2}$ in a 2032 configuration coin cell assembled in an argon-atmosphere glove box. Coin cell details are available in Gilbert et al. ${ }^{27}$ and are also summarized in Table I. The specifics of the assembly process and electrochemical tests performed are also elaborated in detail for the full cell, $\mathrm{LiC}_{6} \mid \mathrm{LiNi}_{0.5044} \mathrm{Co}_{0.1986} \mathrm{Mn}_{0.2970} \mathrm{O}_{2}$ in Gilbert et al. ${ }^{27}$ The NCM523 positive electrode investigated here bears direct resemblance to the

Table I. Cell Parameters.

\begin{tabular}{ccc} 
Parameters & Separator & Cathode (NCM523) \\
\hline$L(\mathrm{~m})$ & $25 \times 10^{-6}$ & $34 \times 10^{-6}$ \\
$\varepsilon(-)$ & 0.390 & 0.335 \\
$\tau(-)$ & 1.601 & 1.727 \\
$\varepsilon_{A M}(-)$ & - & 0.518 \\
$\sigma_{S}(\mathrm{~S} / \mathrm{m})$ & - & 0.04 \\
$c_{e, \text { init }}\left(\mathrm{mol} / \mathrm{m}^{3}\right)$ & & 1200 \\
$S_{C S A}\left(\mathrm{~m}^{2}\right)$ & \multicolumn{3}{c}{$1.6 \times 10^{-4}$} \\
$T(\mathrm{~K})$ & 303.15 \\
$R_{C}(\Omega)$ & \multicolumn{3}{c}{1.5}
\end{tabular}

\section{Table II. Active Material Properties.}

\begin{tabular}{cc} 
Parameters & Values \\
\hline$M M(\mathrm{~g} / \mathrm{mol})$ & 96.56 \\
$c_{s}{ }^{\max }\left(\mathrm{mol} / \mathrm{m}^{3}\right)$ & 48230 \\
$c_{t h}(\mathrm{mAh} / \mathrm{g})$ & 275.62 \\
$V_{m}\left(\mathrm{~cm}^{3} / \mathrm{mol}\right)$ & 20.73 \\
$R_{p r}(\mathrm{~m})$ & $0.5 \times 10^{-6}$ \\
$R_{s c}(\mathrm{~m})$ & $5.3 \times 10^{-6}$ \\
$R_{s}(\mathrm{~m})$ & $5.3 \times 10^{-6}$ \\
$\psi_{\max }$ & 1.841
\end{tabular}

one probed in Gilbert et al., ${ }^{27}$ differing only in the cell configuration (half-cell vs full cell respectively). Table II shows the molar mass, maximum Li concentration and theoretical capacity of NCM523 positive electrode assuming complete delithiation.

GITT tests were performed on NCM523 samples, the electrode parameters and protocol details are furnished in Table III. Two chargedischarge cycles were performed at $\mathrm{C} / 10$ prior to the pulse-rest GITT protocol. A C/20 current pulse was applied for 10.95 minutes for 100 pulses in the voltage range $3.0 \mathrm{~V}-4.4 \mathrm{~V}$. During the pulse period, data is stored at every voltage increment/decrement of $0.001 \mathrm{~V}$ or if maximum time period between successive voltage data exceeds 60 seconds. The rest period between pulses was 120 minutes during charge and 135 minutes during discharge for the GITT experiment. During the rest period, data is stored at every voltage increment/decrement of 0.001 Volts or if maximum time period between successive voltage data is 600 seconds. We have chosen 100 pulses during both charge and discharge to get more accurate open circuit potential (OCP) information for the NCM523 cathode. With 100 pulses, we get a change of state of charge, SOC, of approximately $(1-0.3) / 100=0.0007$ per pulse. Thus, using larger number of pulses generates more data points and can help identify the OCP behavior at all points with less chance of missing any SOC regions which show steep voltage variation. NCM523 exhibits steep voltage change regions close to upper ( $>0.95$ ) limit of state of charge. To quantify that region well, it is necessary to use shorter current pulses toward the end of lithiation.

It is possible to use 25 pulses of longer duration in the GITT test since NCM523 shows fairly smooth voltage variation with state of charge. Close to the end of lithiation, shorter current pulses can be used to identify the state of charge. Use of 40 pulses each during charge and discharge has been demonstrated by Yang et al. ${ }^{22}$ The rest period between charge and discharge pulses is given so that the cell can equilibrate to open circuit conditions. Beyond a threshold rest time value, the cell voltage should become constant with time. The idea behind giving different rest times for charge and discharge was to discern any noticeable open circuit variation with rest period duration. Since, the rest times used are long enough for the cell to equilibrate, we conclude that both the rest times are valid.

Further electrochemical testing was performed to characterize the rate capability of the NCM523 electrodes. The C-rates investigated ranged from $\mathrm{C} / 27$ to $1.5 \mathrm{C}$. It should be duly noted here that charging and discharging correspond to delithiation and lithiation of NCM523, respectively. Conventions dictate that positive electrode materials (e.g. transition metal oxides) always delithiate/lithiate during charge/discharge for both half and full cell configurations.

\section{Table III. GITT Dataset.}

Parameters

$m_{A M}(\mathrm{~g})$

$c_{\text {cell }}(m A h)$

$S_{C S A}\left(\mathrm{~m}^{2}\right)$

Pulse Time (min)

Pulse current (mA)

Rest Time between pulse (min)
100 pulse GITT

0.01363

2.46

$1.58 \times 10^{-4}$

10.95

0.136

120 (Charge), 135 (Discharge) 

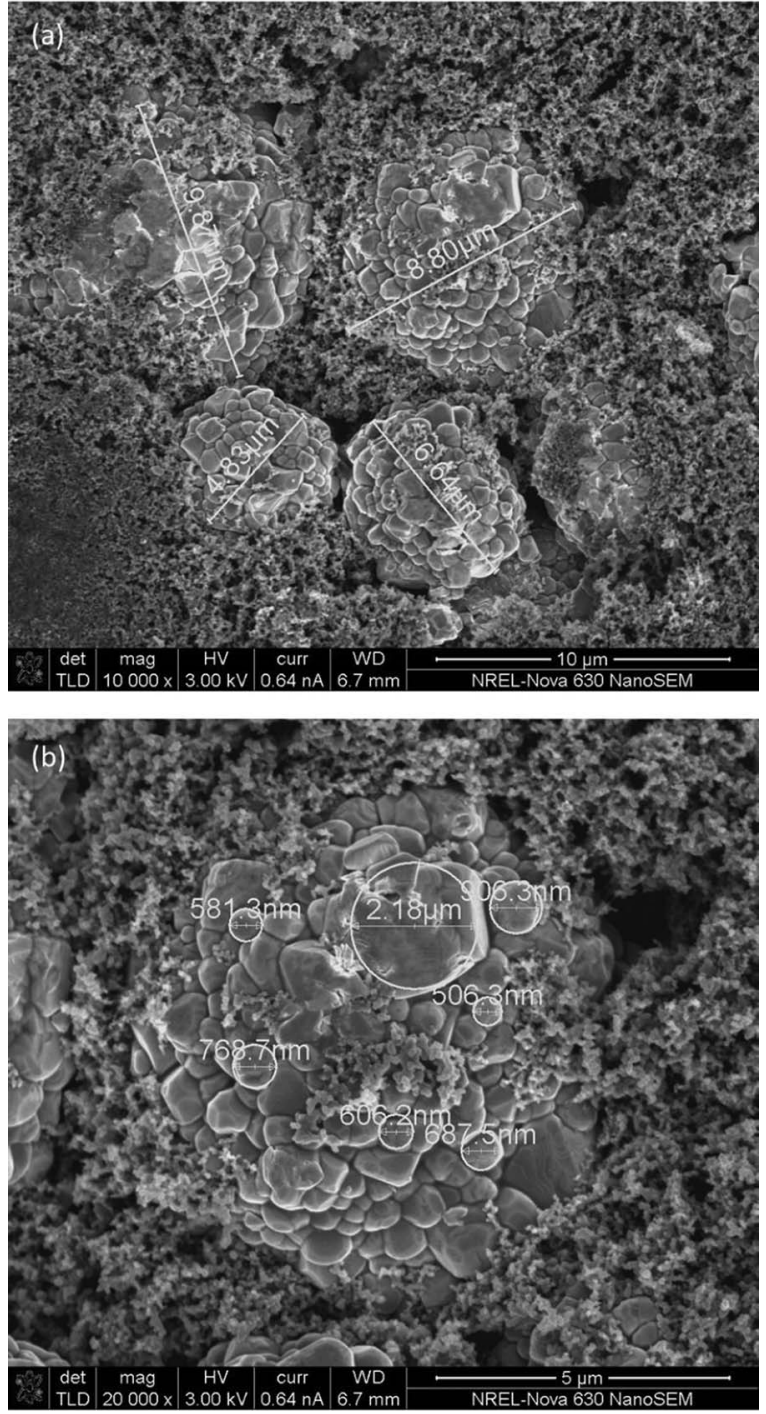

Figure 2. Scanning electron micrograph (SEM) of NCM523 electrode at (a) 10000 and (b) 20000 magnification. The active material exhibits bimodal size distribution, with agglomerate size of the order of $10 \mu \mathrm{m}$ and sub-micron primary particles.

However, for negative electrode materials (e.g. graphite) charge/discharge implies delithiation/lithiation for half-cell and lithiation/delithiation for full cell.

In addition to electrochemical testing, dry NCM523 electrode samples manufactured at ANL were analyzed for their morphology and elemental composition using scanning electron microscopy (SEM) and energy-dispersive X-ray spectroscopy (EDX) respectively at National Renewable Energy Labs (NREL). Figures $2 \mathrm{a}$ and $2 \mathrm{~b}$ show a representative SEM image of the composite NCM523, conductive additive and binder electrode microstructure. It can be readily observed that NCM523 particles exhibit agglomeration to form a spherical secondary unit from sub-micron sized primary elements. Conductive additive and binder form a mesoporous network structure uniformly distributed around the NCM523 agglomerates. The conductive additive used is Timcal C-45 carbon black and binder is SOLVAY Solef 5130 polyvinylidene fluoride (PVDF). Figures $3 \mathrm{a}$ and $3 \mathrm{~b}$ exhibit the elemental spectra from the EDX analysis of the electrode samples. Two different sample regions are investigated; NCM523 agglomerate and conductive additive + binder $(\mathrm{CA}+\mathrm{B})$ porous mesh. An interesting feature that can be observed is the presence of discernible fluorine (F) and aluminum ( $\mathrm{Al}$ ) peaks in addition to the nickel (Ni), cobalt
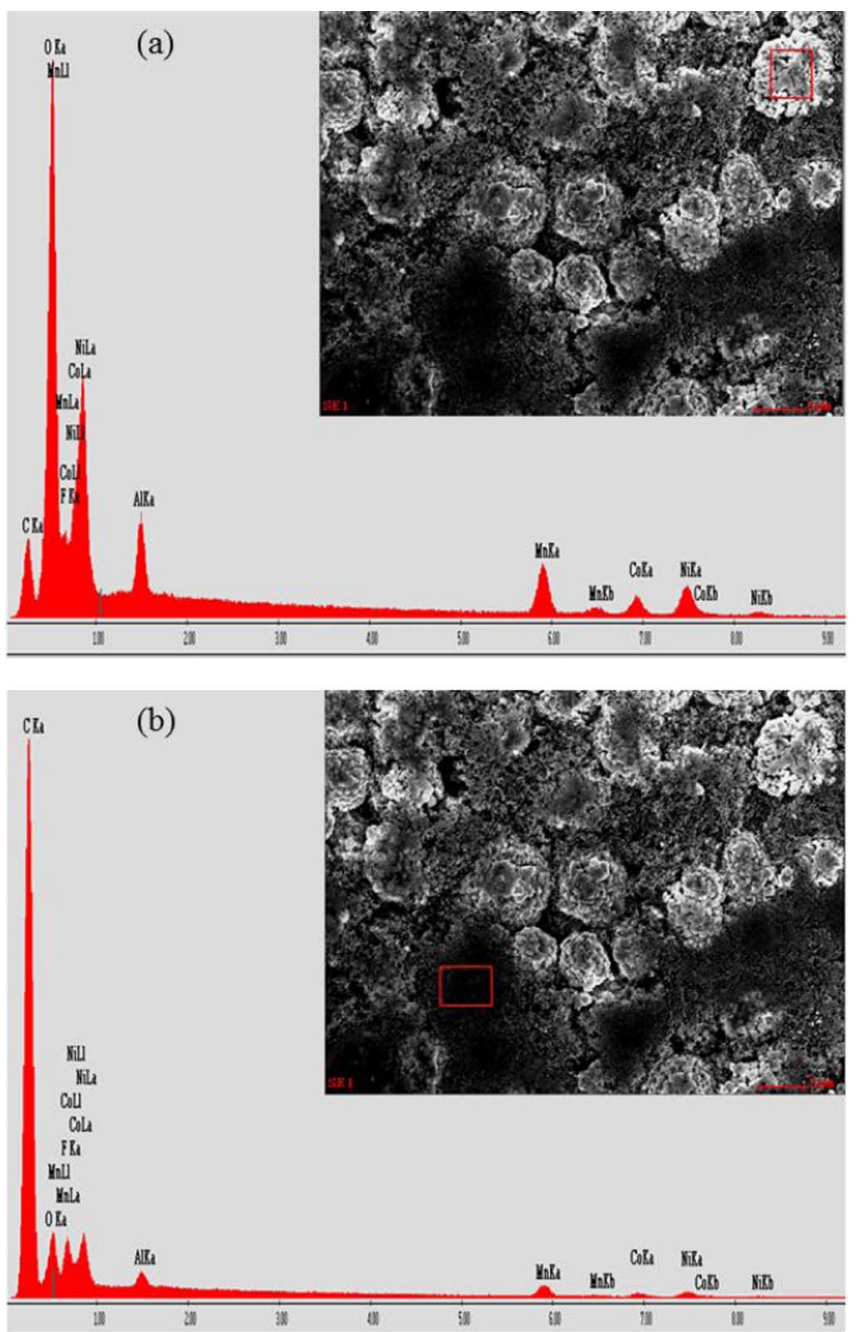

Figure 3. Elemental analysis using energy dispersive X-ray spectroscopy of (a) NCM523 active material agglomerate and (b) conductive additive + binder matrix.

(Co), manganese $(\mathrm{Mn})$, carbon $(\mathrm{C})$ and oxygen $(\mathrm{O})$ elements. Prominence of $\mathrm{Ni}, \mathrm{Co}, \mathrm{Mn}$ and $\mathrm{O}$ in the agglomerate region and $\mathrm{C}$ in $\mathrm{CA}+\mathrm{B}$ region is self-evident. $\mathrm{F}$ atoms are attributed to polyvinylidene fluoride (PVDF) binder while $\mathrm{Al}$ is part of the cathode current collector. No lithium (Li) peaks are observed because of low intensity of generated X-ray signal. ${ }^{28}$

\section{Results and Discussion}

Analysis of experimental GITT dataset.-The GITT dataset can be used to compute solid state diffusion coefficient for $\mathrm{Li}$ inside NCM523 particle as well kinetic rate constant for the Li intercalation reaction. The analysis of GITT datasets for battery materials in literature predominantly report the diffusion coefficient magnitudes while kinetic rate constants are rarely provided. In this section, we will present an exhaustive methodology of analyzing GITT datasets for obtaining both quantities.

Determination of chemical diffusion coefficient.-GITT has primarily been used in battery literature for obtaining the diffusion coefficients for solid-solution intercalation without change of phase. The seminal work on the GITT theory by Weppner and Huggins, ${ }^{29}$ demonstrates its applicability in determining the kinetic parameters for $\mathrm{Li}_{3} \mathrm{Sb}$, a mixed electrode system. The approach is based on one dimensional diffusion of solid atoms into a semi-infinite domain governed by Fick's 
(a)

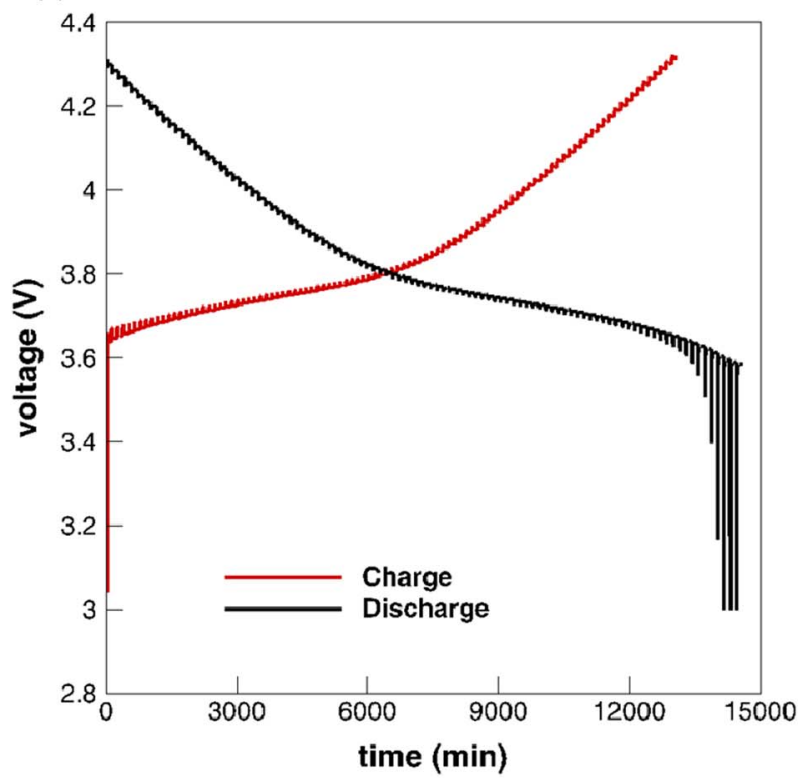

(b)

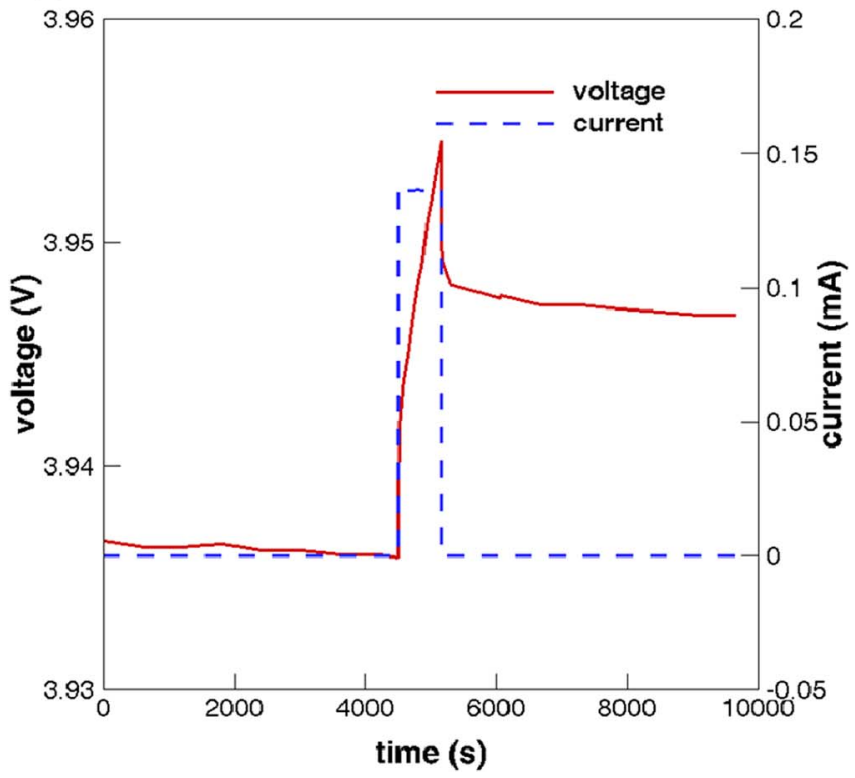

Figure 4. (a) Full 3.0-4.4 V GITT cycle dataset for voltage as function of time. (b) Voltage and current as a function of time for a rest-charge-rest step of the GITT dataset.

law with Nernstian thermodynamics relating the surface concentration to the recorded voltage and current pulse magnitude. For short duration low magnitude current pulses, the above assumption holds true as the Li will not have diffused far from the active material particle surface. Dees et al. ${ }^{30}$ enumerates a succinct list of assumptions necessary for the validity of the GITT model. Recently, GITT theory has also been extended to materials with phase transformation. ${ }^{31}$

Figure 4a shows the GITT cycling voltage vs time data for charge and discharge of the NCM523 electrode material with voltage operating range between $3.0 \mathrm{~V}$ and $4.4 \mathrm{~V}$. The total duration of the experiment is around 460 ( 218 for charge +243 for discharge) hours which is approximately 19 days. The relative ease of performing a single experiment for characterizing battery material properties makes GITT a worthwhile investment although the experiment duration is prolonged. It also makes it imperative to design the experiment with utmost care. Diffusion coefficient, $D_{s}$, can then be extracted from this dataset according to the following equation, ${ }^{29,30}$

$$
D_{s}=\frac{4}{\pi}\left(\frac{I V_{m}}{F S}\right)^{2}\left(\frac{d U^{0} / d y}{d V / d \sqrt{t}}\right)^{2}, \quad t \ll \frac{R^{2}}{D_{s}}
$$

Here, $I$ is current pulse magnitude, $V_{m}$ is NCM523 active material molar volume, $F$ is Faraday's constant, $S$ is composite electrode active area, $U^{0}$ is NCM523 open circuit potential, $y$ is state of charge (SOC), $V$ and $t$ are voltage and time during the current pulse and $R$ is the diffusion length, assumed to be equal to the agglomerate particle radius for NCM523. $I, V_{m}$ and $F$ values are readily available (see Table II). Thus, the unknowns in the above equation are $S, d U^{0} / d y$ and $d V / d \sqrt{t}$. $U^{0}$ vs $y$ is obtained from the steady state voltage of the GITT half-cell during the rest period. Figure $4 \mathrm{~b}$ shows an enlarged single charge step out of the numerous current pulses utilized in the GITT experiment. The charge step is preceded by a rest phase which gives the open circuit voltage at the previous step. During the current pulse, we can identify two regimes of voltage increase. There is an abrupt jump in voltage during a short time period. This can be attributed to the ohmic and kinetic overpotentials which are characterized by fast response times. The latter period of current pulse exhibits a steady increase in voltage in synchronization with the concentration overpotential due to solid state diffusion which is a slower process. After application of the current pulse, the NCM523 electrode is delithiated by a fixed amount. The new SOC can be calculated from the measured charge passed while $U^{0}$ at the new SOC is given by the voltage at the end of the following rest period.

The voltage curve in Figure 4a looks noisy because it shows voltage response during all current pulses and rest over the entire course of the GITT experiment. As can be seen in Figure $4 b$, one single current pulse preceded and succeeded by rest phase shows step change in voltage. If we extend this behavior over 100 pulses, the voltage response will look like the one shown in Figure 4a. No voltage filtering techniques are used. From Figure 4b, it can also be seen that voltage is still trending downward during the rest period before the pulse. However, the decay rate is very small and the voltage usually stabilized for most of the pulses. Voltage behavior is also a bit erratic after the pulse due to initial sharp change and then stabilizes slowly.

Accurate computation of SOC at the end of each current pulse necessitates precise estimation of SOC at the beginning of the charging process. Inductively coupled plasma optical electron microscopy (ICP/OES) has been used in literature to identify the initial stoichiometry for $\mathrm{Li}$ intercalated cathode active materials. ${ }^{23}$ In its absence, we set the fully intercalated Li stoichiometry (i.e. $y=1$ ) to the start of GITT voltage $(3.0 \mathrm{~V})$, while simultaneously acknowledging reference values available in literature. ${ }^{22}$ Subsequent SOC values are calculated using the current pulse, duration, active material mass and theoretical capacity magnitudes (see Tables II and III). For the 100 pulse GITT data set, a current of $0.136 \mathrm{~mA}$ is applied for a duration of 10.95 minutes. This corresponds to a specific capacity change,

$$
\Delta c=-\frac{I \times t}{m_{A M}}=-\frac{0.136 \times(10.95 / 60)}{0.01363}=-1.8210 \mathrm{mAh} / \mathrm{g}
$$

The negative sign is because of delithiation occurring during charge. Thus, the new state of charge is,

$$
y^{p+1}=y^{p}+\Delta c / c_{t h}=1.0-(1.8210 / 275.6219)=0.9934
$$

This process is continued until we reach the end of the charging process, where the calculated value of $y$ equals 0.3439 . The ensuing discharge step is associated with the lithiation of the active material, thus the SOC will now increase with time. Thus, change in specific capacity will be positive during the discharge process. For our GITT dataset, discharging ends at $y$ equals 0.9915 .

Irreversible lithium loss is a predominant phenomenon due to SEI formation on anode in full cells resulting in depletion of a fixed lithium inventory. Since, in half cells lithium metal anode is used we had assumed that any lithium inventory loss due to SEI formation on anode is circumvented by the presence of excess lithium source on the anode side. Thus, we neglected any irre- 
versible lithium or site loss in the estimate of stoichiometry. However, from charge discharge dataset prior to the onset of GITT measurements, an estimate for the irreversible site loss can be made. The first charge (delithiation) of NCM523 yielded a charge capacity of $203 \mathrm{mAh} / \mathrm{g}$ corresponding to a change of state of charge variation of $203.00 / 275.62=0.7365$. The first discharge (lithiation) of NCM523 yielded a discharge capacity of $180 \mathrm{mAh} / \mathrm{g}$ corresponding to a state of charge of $180.00 / 275.62=0.6531$. This can be construed as an irreversible site loss of $0.7365-0.6531=0.0834$. This loss has not been accounted for in our results. The open circuit potential profile without accounting for the irreversible loss showed good match with existing data reported by Yang et $\mathrm{al}^{22}$ and has hence been used for any further computations (see Figure 15). Incorporation of irreversible site loss data will result in shifting of the open circuit potential curve to the left in the state of charge range $0.26-0.92$.

Figure 5a shows the experimentally computed $U^{0}$ vs $y$ values for both charge and discharge. Calculation of $d U^{0} / d y$ is required for diffusion coefficient estimation. Consequently, we fit a nonlinear equation to the experimentally computed dataset which is also shown in Figure 5a. The equation fit is an important step as numerical differentiation of discrete dataset can lead to spurious errors in the derivative calculation. The use of MATLAB curve fitting routines have been used to best fit the voltage data. The equations that have been fit to the charge and discharge open circuit potentials are detailed here:

$$
\begin{aligned}
U_{C}^{0}= & 4851.489856\left(y^{9}\right)-30525.40243\left(y^{8}\right)+83781.31058\left(y^{7}\right) \\
& -131496.4647\left(y^{6}\right)+129923.6915\left(y^{5}\right)-83737.64466\left(y^{4}\right) \\
& +35194.22436\left(y^{3}\right)-9301.7411\left(y^{2}\right)+1401.288467\left(y^{1}\right) \\
& -87.11977805-0.0003 \exp \left(7.657\left(y^{115}\right)\right)
\end{aligned}
$$

$$
\begin{aligned}
U_{D}^{0}= & 5744.862289\left(y^{9}\right)-35520.41099\left(y^{8}\right)+95714.29862\left(y^{7}\right) \\
& -147364.5514\left(y^{6}\right)+142718.3782\left(y^{5}\right)-90095.81521\left(y^{4}\right) \\
& +37061.41195\left(y^{3}\right)-9578.599274\left(y^{2}\right)+1409.309503\left(y^{1}\right) \\
& -85.31153081-0.0003 \exp \left(7.657\left(y^{115}\right)\right)
\end{aligned}
$$

A $9^{\text {th }}$ degree polynomial fits the open circuit potential data points from $3.6 \mathrm{~V}$ to $4.4 \mathrm{~V}$ and the steep decline from $3.6 \mathrm{~V}$ to $3.0 \mathrm{~V}$ can be mimicked with the help of the exponential function. Figure $5 \mathrm{~b}$ shows the obtained $d U^{0} / d y$ values vs state of charge for both charge and discharge.

Some hysteresis can be observed between the charge and discharge open circuit potentials in Figure 5a. Experiments done by Yang et al. ${ }^{22}$ also exhibit hysteresis in the open circuit potential dataset during charge and discharge. Structural changes and phase transition can contribute to hysteresis between charge and discharge OCPs. Researchers have proposed a reversible structural phase transition or order-disorder transition in oxide cathodes during cycling to hysteresis in OCP profiles but it has not been definitely proven. ${ }^{32-34}$ In operando energy dispersive X-ray diffraction (EDXRD) analysis of NCM523 electrodes during electrochemical charge/discharge is necessary to investigate the cause of this voltage hysteresis.

Self-discharge of the coin cells should not be a probable cause for voltage hysteresis since continuous voltage change was not observed in the voltage data during the rest period for majority of the pulses. The voltage usually stabilized during the nearly two-hour long rest period after the current pulses and remained constant. If self-discharge was occurring, we would have seen continuous change in the voltage data throughout the rest period without any stabilization. Hence selfdischarge can be ruled out as a possible cause for the hysteresis.

The remaining unknowns to be determined in the Equation 1 are $S$ and $d V / d \sqrt{t}$. Figure 6 a shows the $V$ vs $\sqrt{t}$ curve for a single current pulse. GITT theory suggests a linear trend line for this plot beyond a threshold time (10-100 seconds) due to the existence of concentration overpotential. ${ }^{29}$ The initial voltage rise associated with ohmic and

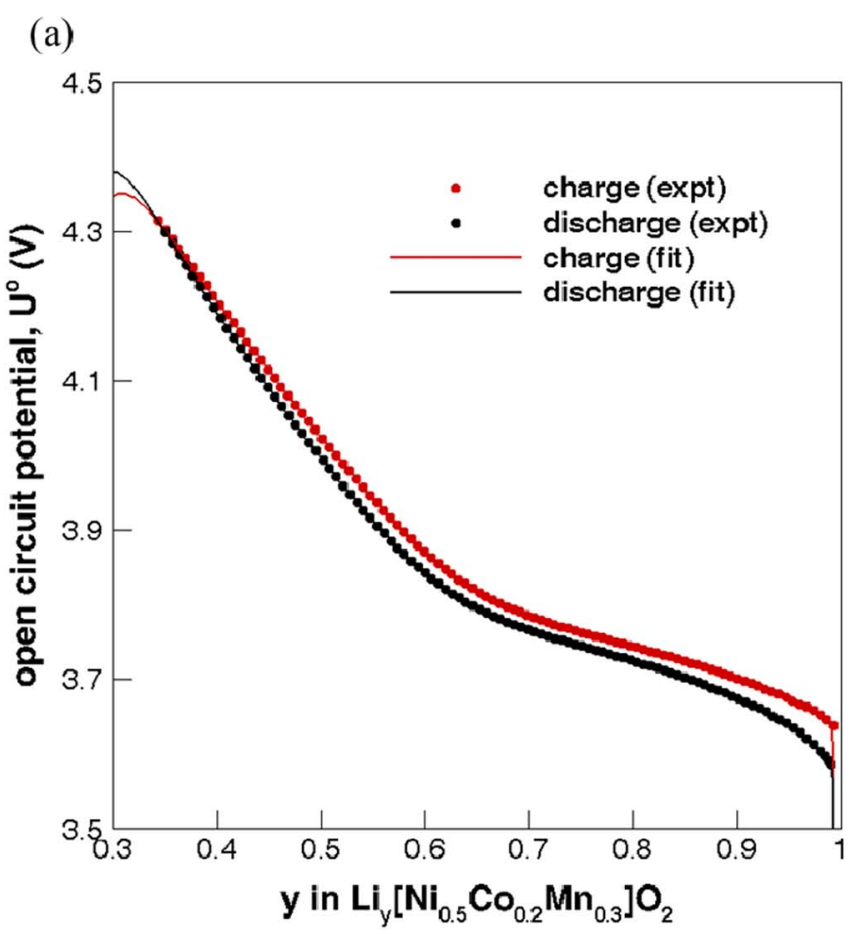

(b)

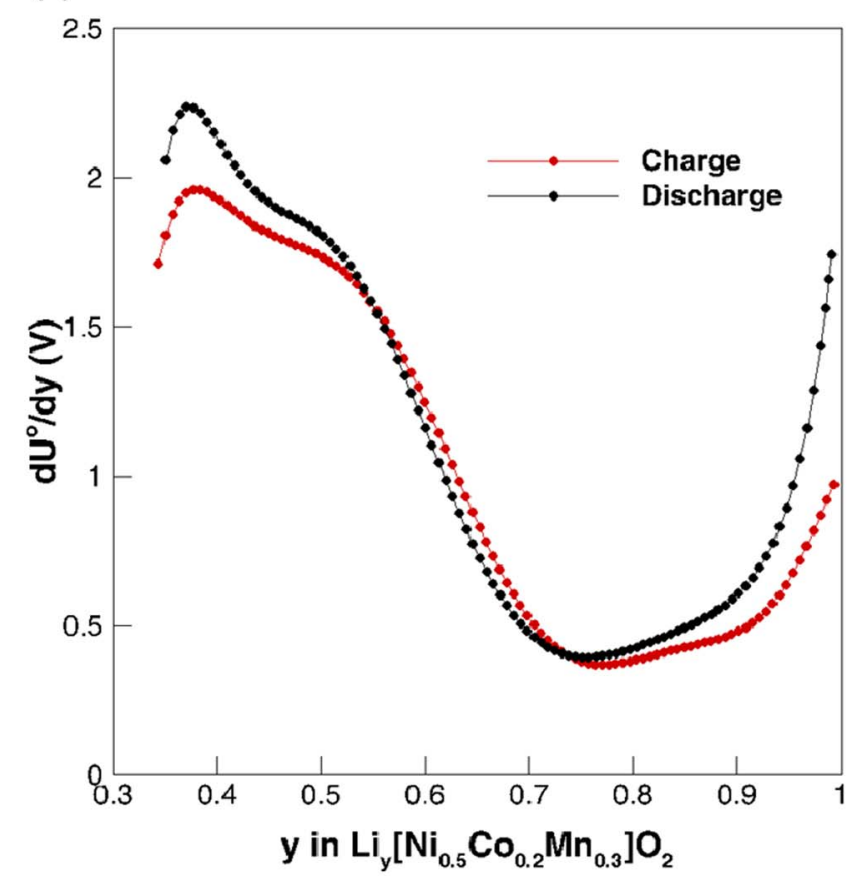

Figure 5. (a) Open circuit potential $\left(U^{0}\right)$ of NCM523 as a function of state of charge with the nonlinear fit curves. (b) Derivative of equilibrium potential as a function of state of charge.

charge transfer overpotential shows nonlinear trend with $\sqrt{t}$. Thus, $d V / d \sqrt{t}$ can be computed by excluding the first few voltage data points $(t<25$ seconds) during the current pulse and obtaining a linear fit for the remaining points. The threshold time for our analysis is selected through visual inspection of the $V$ vs $\sqrt{t}$ curve beyond which linear behavior is observed. This process is repeated for all the 100 charge and 100 discharge pulses and the final computed values are shown in Figure $6 \mathrm{~b}$. The minimum $\mathrm{R}^{2}$ value accepted over all the points is 0.99 . For the voltage range 3.6 to $4.4 \mathrm{~V}$, the $\mathrm{R}^{2}$ is approxi- 
(a)

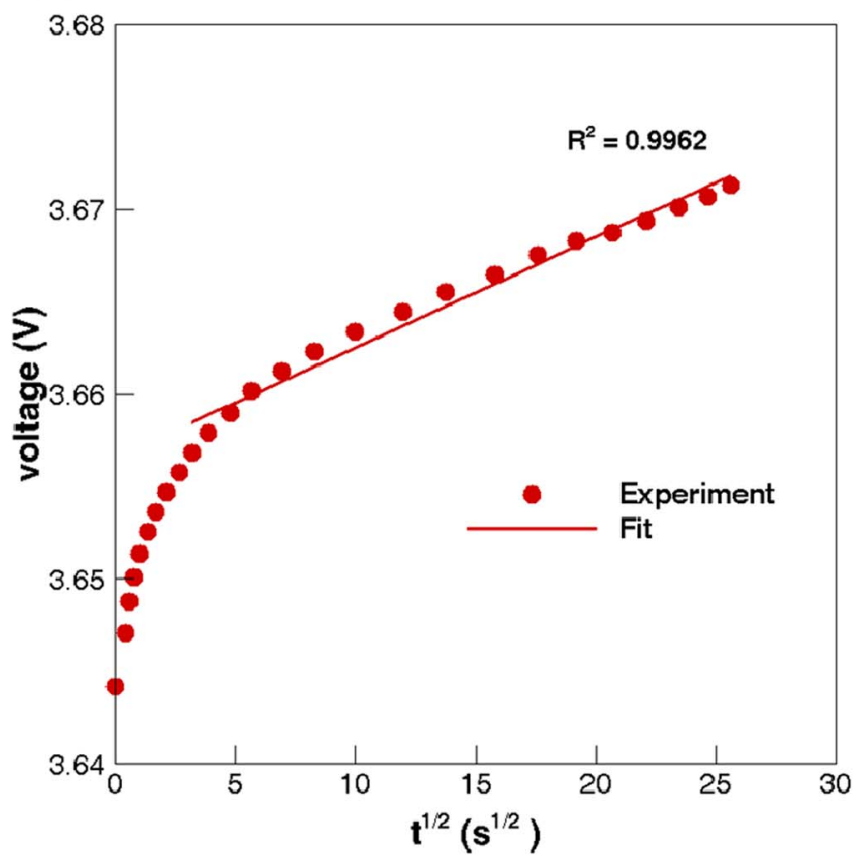

(b)

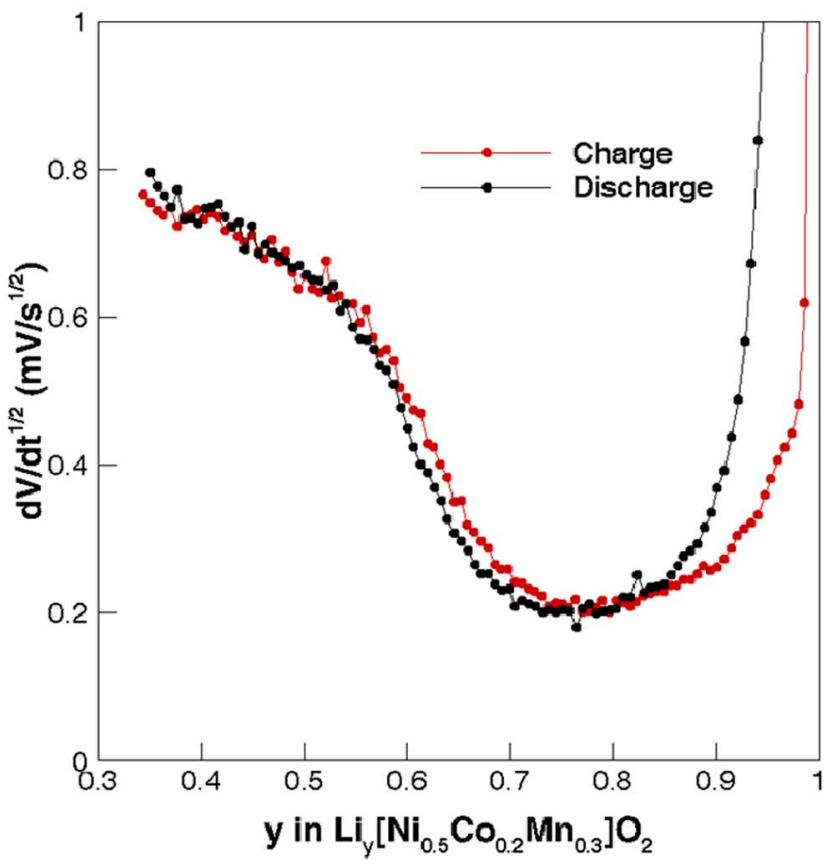

Figure 6. (a) Voltage as a function of square root of time for a single charging current pulse duration with the corresponding linear fit (b) Slope of voltage vs square root of time for the full GITT dataset.

mately close to 0.995 . Only close to the end of lithiation, due to steep change in voltage ( 3.0 to $3.6 \mathrm{~V}$ ) the $\mathrm{R}^{2}$ value goes close to 0.99 .

Since the calculation of active area, $S$, is a non-trivial exercise we formulate an area independent formulation for diffusivity. This is accomplished by taking the area term to the left hand side of Equation 1. The corresponding diffusivity relation is given by:

$$
S^{2} D_{s}=\frac{4}{\pi}\left(\frac{I V_{m}}{F}\right)^{2}\left(\frac{d U^{0} / d y}{d V / d \sqrt{t}}\right)^{2}
$$

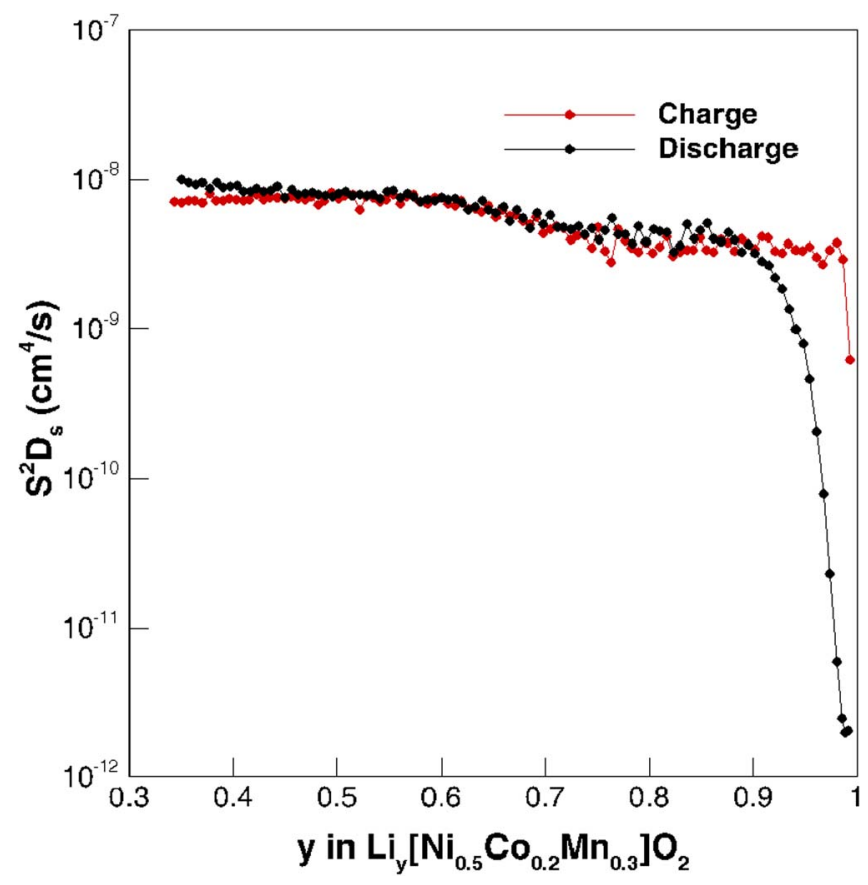

Figure 7. Area independent diffusivity as a function of state of charge obtained from the full GITT dataset.

Figure 7 gives the variation of $S^{2} D_{s}$ vs $y$ for both charge and discharge. This state of charge variation of $S^{2} D_{s}$ is further incorporated in our simulation models. The benefits of formulating an area independent diffusivity are discussed in a subsequent section on active area estimation. Accurate values of interfacial area are required for calculation of all the characteristic transport and kinetic properties. Hence, we propose to use this area as a parameter we estimate in our models and hope to ascertain its value based on maximum congruence between the experimental and simulation results.

Determination of Li intercalation rate constant.-Charge transfer kinetics characterization can also be achieved by direct analysis of the experimental GITT dataset. We propose the following method backed by reasonable assumptions to estimate the state of charge dependent charge transfer resistance, exchange current density and $\mathrm{Li}$ intercalation rate constant for Li ion battery materials using GITT. We will again keep the formulations independent of area which will be incorporated later.

The current used in our GITT experiments is $0.136 \mathrm{~mA}$ with coin cell discharge capacity of $2.46 \mathrm{mAh}$ for cycling between $3.0-4.4 \mathrm{~V}$ (see Table III). This corresponds to a C-Rate of C/18.08 which is in the low range. For low current densities, the linear regime approximation of the Butler-Volmer (BV) equation becomes applicable. Thus, accurate estimation of charge transfer overpotential can be used for computing the charge transfer resistance and subsequent exchange current density calculations.

From Figure $4 \mathrm{~b}$ which shows the single step pulse during charge for GITT dataset, we can observe the near vertical rise in voltage for an infinitesimal time at the beginning of the current pulse. This rise, as discussed earlier, is the contribution of ohmic and charge transfer overpotentials. We can ascertain the magnitude of this rise from the GITT datasets for each state of charge. For low current density operation, concentration gradients inside the electrolyte phase and potential gradients in both solid and electrolyte phase are negligible. Ohmic overpotential from direct electrode resistance which is 8.5 $\Omega-\mathrm{cm}^{2}$ results in $0.731 \mathrm{mV}$ ohmic contribution while the total rise is around $50 \mathrm{mV}$. Direct electrode resistance is computed using the electronic conductivity measurement and dimensions of the electrode, 
given in Equation 7.

$$
R_{d c}=\frac{L}{\sigma_{e f f}}=\frac{34 e-4(\mathrm{~cm})}{4 e-4(\mathrm{~S} / \mathrm{cm})}=8.5 \Omega-\mathrm{cm}^{2}
$$

Effective electronic conductivity of the electrode has been found out using direct current measurements on the dry electrode. Consequently, ohmic overpotential due to species and charge transport is small. Thus, we make the approximation of neglecting the contribution of ohmic overpotential to the steep voltage change at the start of current pulse. The voltage change is assumed to be the charge transfer overpotential $\eta_{C T}$ entirely and is given by the Butler-Volmer (BV) current density formulation shown in Equation 8.

$$
i=\frac{I}{S}=i_{0}\left[\exp \left(\frac{\alpha_{a} F}{\Re T} \eta_{C T}\right)-\exp \left(-\frac{\alpha_{c} F}{\Re T} \eta_{C T}\right)\right]
$$

Linearization of Butler Volmer for small current densities leads to Equation 9.

$$
i=\frac{I}{S}=i_{0} \frac{\left(\alpha_{a}+\alpha_{c}\right) F}{\Re T} \eta_{C T}=i_{0} \frac{F}{\Re T} \eta_{C T}
$$

Further rearrangements lead to formulation of area independent charge transfer resistance and exchange current density in Equations 10, 11 and 12. The initial voltage rise during GITT current pulse is used to calculate $\eta_{C T}$. Area independent charge transfer resistance and exchange current density can then be computed by substituting the current $(I=0.136 \mathrm{~mA})$, temperature $(T=303.15 \mathrm{~K})$, universal gas constant $(R=8.314 \mathrm{~J} / \mathrm{mol}-\mathrm{K})$ and Faraday's constant $(F=96485.33 \mathrm{C} / \mathrm{mol})$.

$$
\begin{gathered}
R_{C T}=\frac{\eta_{C T}}{i}=\frac{\Re T}{i_{0} F} \\
\frac{R_{C T}}{S}=\frac{\eta_{C T}}{I}=\frac{\Re T}{i_{0} S F} \\
\frac{R_{C T}}{S}=\frac{\eta_{C T}}{I}, i_{0} S=\frac{\Re T}{F} \frac{I}{\eta_{C T}}
\end{gathered}
$$

The final computation is that of the area independent Li intercalation rate constant ${ }^{35}$ as shown in Equations 13, 14 and 15. The computations are done for different states of charge for all the 100 charge and 100 discharge pulses of GITT dataset.

$$
\begin{gathered}
i_{0}=k F c_{s}{ }^{\max } c_{e}{ }^{0.5}\left(1-\theta_{\text {surf }}\right)^{0.5} \theta_{\text {surf }}{ }^{0.5} \\
i_{0} S=k S F c_{s}{ }^{\max } c_{e}{ }^{0.5}\left(1-\theta_{\text {surf }}\right)^{0.5} \theta_{\text {surf }}{ }^{0.5} \\
k S=\frac{i_{0} S}{F c_{s}{ }^{\text {max }} c_{e} e^{0.5}\left(1-\theta_{\text {surf }}\right)^{0.5} \theta_{\text {surf }} .5}
\end{gathered}
$$

Figures $8 \mathrm{a}$ and $8 \mathrm{~b}$ shows the area independent charge transfer resistance and exchange current density variation with state of charge for NCM523 electrode. Figure 9 gives the area independent $\mathrm{Li}$ intercalation rate constant for NCM523 and its variation with state of charge.

Estimation of active area.-The accurate estimation of interfacial area is imperative for calculations of diffusivity, charge transfer resistance and $\mathrm{Li}$ intercalation rate constant for the active material as explained before. As shown in Figure 2, the $\mathrm{LiNi}_{0.5044} \mathrm{Co}_{0.1986} \mathrm{Mn}_{0.2970} \mathrm{O}_{2}$ active material shows bimodal structural features comprising of secondary particles with dimension of the order of $10 \mu \mathrm{m}$. These secondary particles further contain several primary particles in the submicron range. The evaluation of active area requires consideration of this bimodal distribution. In literature, active area computations

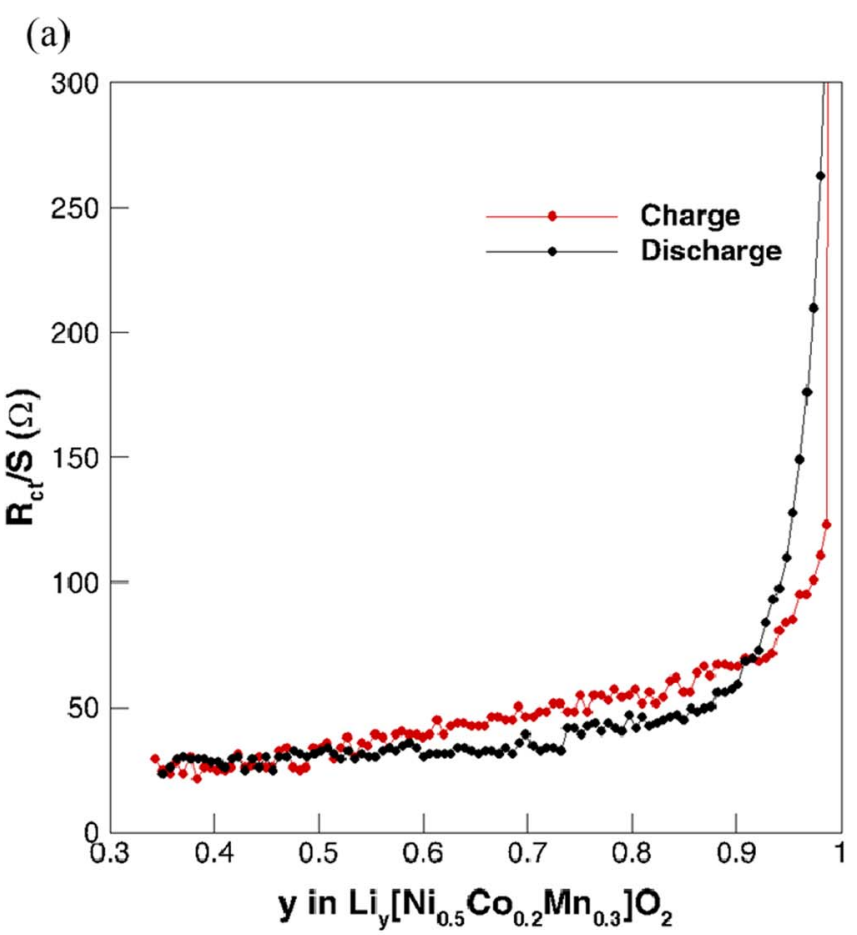

(b)

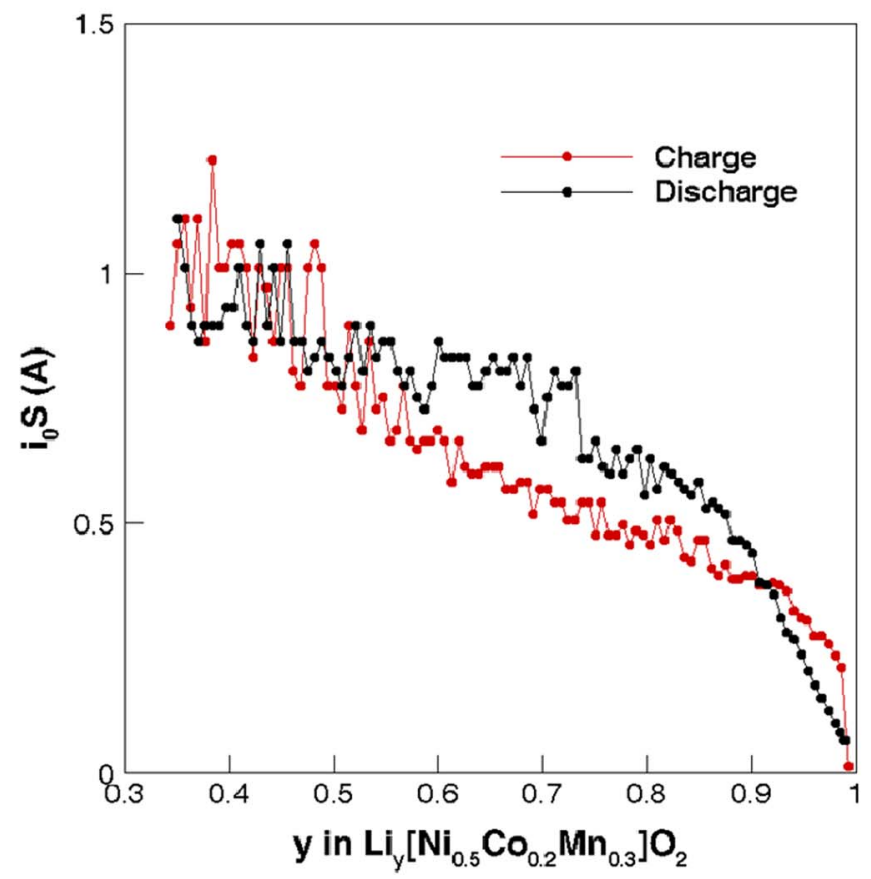

Figure 8. (a) Area independent charge transfer resistance and (b) area independent $\mathrm{Li}$ intercalation exchange current density as a function of state of charge calculated from the full GITT dataset.

for the macro homogeneous models involves the assumption of nonoverlapping spherical particles inside the electrode medium. We extend this model to account for the agglomeration of primary particles into a secondary particle.

Firstly, we introduce the derivation of active area considering unimodal spherical particles commonly utilized in literature. The subsequent calculation of active area for agglomerate particles will mimic this methodology. The computation steps are enumerated as follows: 


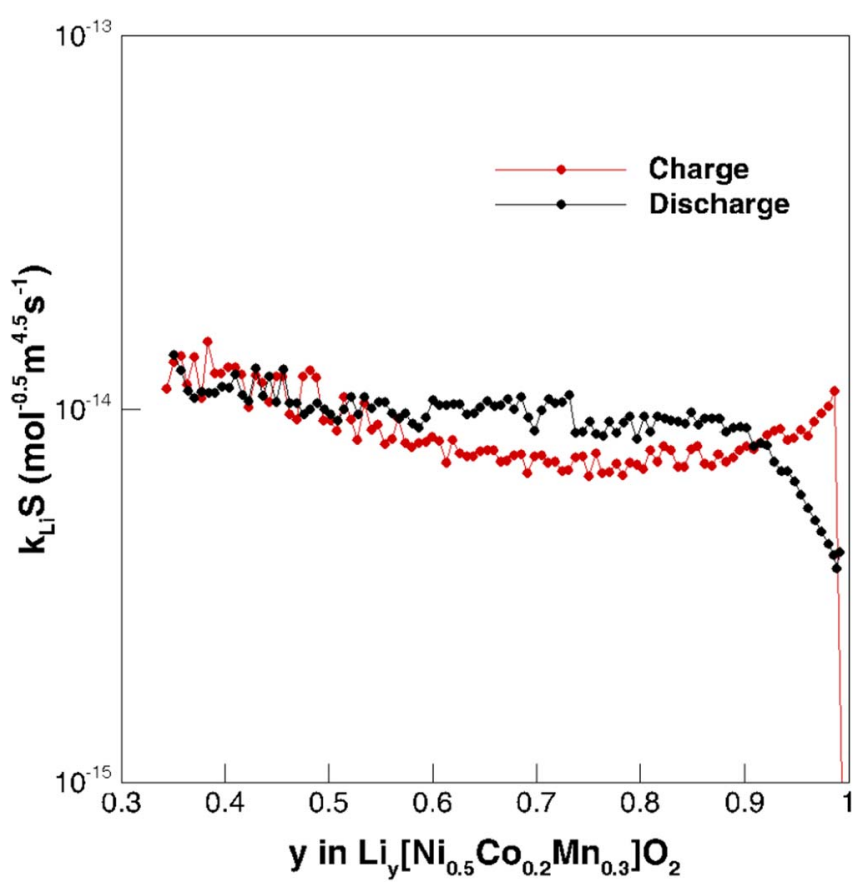

Figure 9. Area independent $\mathrm{Li}$ intercalation rate constant as a function of state of charge computed from the full GITT dataset.

Total active material volume inside cathode is given by the active material volume fraction and cathode volume.

$$
V_{A M}=\varepsilon_{S} V_{c a t}=\varepsilon_{S} S_{c s a} L_{c a t}
$$

Volume and surface area of a spherical particle are given by

$$
V_{s}=\frac{4}{3} \pi R^{3}, \quad S_{s}=4 \pi R^{2}
$$

Thus, the total number of spherical particles inside electrode can be calculated as

$$
N_{s}=\frac{V_{A M}}{V_{s}}=\frac{\varepsilon_{S} S_{c s a} L_{c a t}}{\frac{4}{3} \pi R^{3}}
$$

Consequently, total active area inside electrode is given by

$$
S_{A M}(\text { sphere })=N_{s} \times S_{s}=\frac{3 \varepsilon_{S}}{R_{s}} S_{c s a} L_{c a t}
$$

The agglomerate structure is presumed to be a spherical secondary particle overlapped by hemispherical primary particles as shown in Figure 10 . Radius of the secondary and primary particles are denoted by $R_{s c}$ and $r_{p r}$ respectively. The number of primary particles that can overlay this secondary structure is determined utilizing the projected area of the diminutive primary particle on the relatively larger secondary particle. Projected area for a hemisphere is given by

$$
S_{p r o j}=\pi r_{p r}^{2}
$$

Thus, number of primary particles surrounding the primary particle can then be computed as,

$$
N_{p r}=\frac{S_{s c}}{S_{p r o j}}=\frac{4 \pi R_{s c}^{2}}{\pi r_{p r}^{2}}
$$

Going further, volume and surface area for this agglomerate structure comprising of 1 spherical secondary particle and $N_{p r}$ hemispherical primary particles is required. Volume calculation requires the straightforward addition of the volumes of the constituent elements

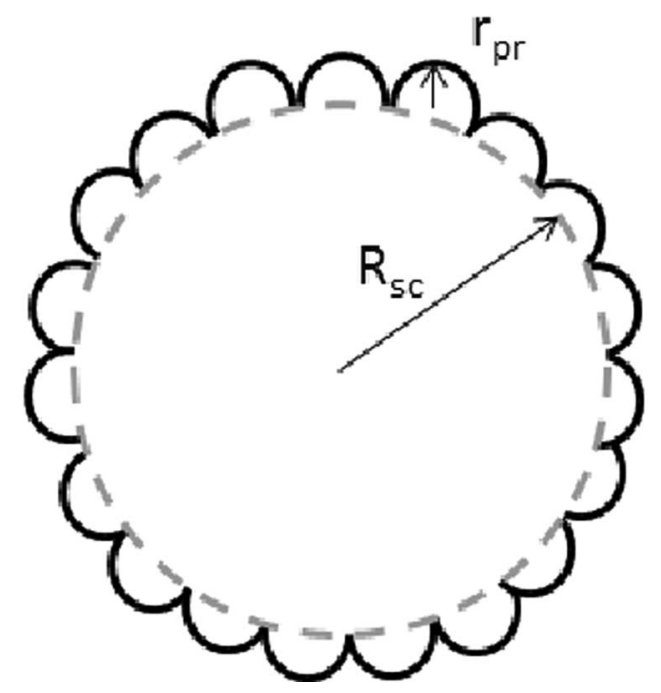

Figure 10. Schematic representation of spherical NCM523 secondary agglomerates of radius $R_{s c}$ with hemispherical primary particles of radius $r_{p r}$ overlaying on top of the secondary structure.

and is given by,

$$
\begin{aligned}
V_{a g g} & =1 \times \frac{4}{3} \pi R_{s c}^{3}+N_{p r} \times \frac{2}{3} \pi r_{p r}^{3}=\frac{4}{3} \pi R_{s c}^{3}+\frac{4 \pi R_{s c}^{2}}{\pi r_{p r}^{2}} \times \frac{2}{3} \pi r_{p r}^{3} \\
& =\frac{4 \pi}{3}\left(R_{s c}^{3}+2 R_{s c}^{2} r_{p r}\right)
\end{aligned}
$$

Agglomerate surface area computation requires summing up the contributions of the $N_{p}$ hemispherical primary elements and is given by,

$$
S_{a g g}=N_{p r} \times 2 \pi r_{p r}^{2}=\frac{4 \pi R_{s c}^{2}}{\pi r_{p r}^{2}} \times 2 \pi r_{p r}^{2}=8 \pi R_{s c}^{2}
$$

The total electrode active area can now be calculated by counting the gross number of agglomerate units inside the electrode and multiplying by the surface area of individual agglomerate. Total count of agglomerates inside the electrode volume is

$$
N_{a g g}=\frac{V_{A M}}{V_{a g g}}=\frac{\varepsilon_{S} S_{c s a} L_{c a t}}{\frac{4 \pi}{3}\left(R_{s c}^{3}+2 R_{s c}{ }^{2} r_{p r}\right)}
$$

Finally, total electrode active area can be computed using,

$$
\begin{aligned}
S_{A M}(\text { agglomerate }) & =\frac{\varepsilon_{S} S_{c s a} L_{c a t}}{\frac{4 \pi}{3}\left(R_{s c}^{3}+2 R_{s c}^{2} r_{p r}\right)} \times 8 \pi R_{s c}^{2} \\
& =\frac{6 \varepsilon_{S}}{R_{s c}+2 r_{p r}} S_{c s a} L_{c a t}
\end{aligned}
$$

The above exercise is important as it delineates the difference in active area caused by particle morphology. The bimodally distributed $\mathrm{LiNi}_{0.5044} \mathrm{Co}_{0.1986} \mathrm{Mn}_{0.2970} \mathrm{O}_{2}$ particle exhibits significantly altered active area as compared to a simple unimodal spherical morphology. This can be further analyzed by computing the active area ratios for the agglomerate element electrode to spherical element electrode. This is given by,

$$
\psi=\frac{S_{A M}(\text { agglomerate })}{S_{A M}(\text { sphere })}=\frac{\frac{6 \varepsilon_{S}}{R_{s c}+2 r_{p r}} S_{c s a} L_{c a t}}{\frac{3 \varepsilon_{S}}{R_{s}} S_{c s a} L_{c a t}}=\frac{2 R_{s}}{R_{s c}+2 r_{p r}}
$$

Using values of $R_{s c}=5 \mu \mathrm{m}, r_{p r}=0.5 \mu \mathrm{m}$ and assuming a spherical particle of radius equivalent to center to tip distance of the agglomerate, i.e., $R_{s}=R_{s c}+R_{p r}$ gives us an active area ratio, $m=1.833$. A large increase in active area magnitude is obtained 
for agglomerate active particles as compared to spherical particles. Thus, computation of essential kinetic and transport parameters for $\mathrm{LiNi}_{0.5044} \mathrm{Co}_{0.1986} \mathrm{Mn}_{0.2970} \mathrm{O}_{2}$ material necessitates accounting for this large variability in active area. Recent battery literature devoted to characterization of materials similar to $\mathrm{LiNi}_{0.5044} \mathrm{Co}_{0.1986} \mathrm{Mn}_{0.2970} \mathrm{O}_{2}$ is devoid of discussions related to aforementioned issues. In general, area calculation is attempted using the aforementioned simplistic spherical particle formulation, analysis of 3D volume data from stochastically generated image based (XCT/FIB-SEM) reconstructed microstructures ${ }^{35}$ or using experimental techniques like BET (Brunauer-Emmett-Teller) ${ }^{36}$ characterization involving adsorption of gas molecules on the electrode surface. Spherical active material particle formulation underestimates the active area for agglomerated structures as demonstrated previously. Stochastic/image based reconstruction provide accurate volume data for characterization of active area but are time consuming and expensive. The BET technique is also contentious as it overestimates the active surface area for composite electrodes. Owing to the poor intrinsic electronic conductivity of the transition metal oxide cathode intercalation materials, an amalgamation of conductive additive and binder is utilized to circumvent charge transport limitations inside the solid phase to enhance battery performance. This electrochemically inert component aimed at providing electronic pathways also coats portion of the active metal oxide surface, effectively rendering the coating area inactive. Consequently, BET experiments may not be representative of the true area of the active phase within the composite electrode because gas adsorption occurs at both active and inactive sites.

Utilizing GITT, half-cell performance datasets and the agglomerate area formulation, it is possible to extract the active area for $\mathrm{LiNi}_{0.5044} \mathrm{Co}_{0.1986} \mathrm{Mn}_{0.2970} \mathrm{O}_{2}$ electrodes using macrohomogeneous LIB models. The macrohomogeneous single particle (SPM) ${ }^{37,38}$ and pseudo $2 \mathrm{D}$ models $(\mathrm{P} 2 \mathrm{D})^{7}$ are retrofitted allowing for active area variation. The formulation of both these models traditionally hinges on the spherical particle assumption. Consequently, our model formulation incorporates this while introducing an additional surface roughness $(\psi)$ parameter to account for active area change as compared to spherical unimodal particles. The active area in our model is given by

$$
S_{A M}=\frac{3 \varepsilon_{S}}{R_{s}} S_{c s a} L_{c a t} \times \psi
$$

The search space for surface roughness $(\psi)$ is bounded using the active area formulation we defined earlier. The lower bound is dictated by the simple sphere assumption giving $\psi=1$ while the upper bound is governed by the agglomerate to spherical active area ratio given by Equation 26.

The mean particle radius for Li diffusion computation is then computed using,

$$
R_{\text {mean }}=\frac{3 \varepsilon_{S}}{S_{A M}} S_{c s a} L_{c a t}=\frac{R_{s}}{\psi}
$$

The value of $R_{s}$ used is from the average oxide particle diameter from the particle size distribution analysis done using laser diffraction measurement which is reported to be $10.6 \mu \mathrm{m}$. So, $R_{s}$ equals $5.3 \mu \mathrm{m}$.

Surface roughness including performance model.-The application of both single particle and pseudo 2D electrochemical model are presented to emphasize the strengths and accuracy of each approach. In addition, agreement of results obtained from both methods help ascertain the validity of our conclusions. The governing equations for both models are readily available in literature and are summarized in Santhanagopalan et al. ${ }^{38}$

Butler-Volmer kinetics is used to describe the lithium intercalation reaction at both the anode and cathode (Equations 29-33). For the halfcell configuration, anode solid phase potential can be set to zero (vs Li) because of $\mathrm{Li}$ metal anode. ${ }^{39} \mathrm{Li}$ metal anode has open circuit potential of $0 \mathrm{~V}$ vs Li and high magnitude of exchange current density (39400 $\mathrm{A} / \mathrm{m}^{2}$ ), so the resulting overpotential at anode will be negligible.

The performance model also includes cell contact resistance, $R_{c}$, as a tunable parameter to better mimic the experimental GITT and rate performance of the electrodes. Since, the effect of film resistance is similar to that of cell contact resistance in producing a voltage drop/rise we have tried to minimize the number of estimable parameters by including the effect of all resistances into a single term, the cell contact resistance. In this way, the effect of resistive films on lithium counter electrode has also been incorporated into the cell contact resistance term.

$$
\begin{gathered}
i=i_{0}\left[\exp \left(\frac{\alpha_{a} F}{\Re T} \eta\right)-\exp \left(-\frac{\alpha_{c} F}{\Re T} \eta\right)\right] \\
\eta=\phi_{s}-\phi_{e}-U\left(\theta_{s}, T\right) \\
i_{0}=k(T) F c_{s}^{\text {max }} c_{e}^{0.5}\left(1-\theta_{\text {surf }}\right)^{0.5} \theta_{\text {surf }}{ }^{0.5} \\
\theta_{\text {surf }}=\frac{c_{s}\left(r=R_{s}\right)}{c_{s}{ }^{\text {max }}} \\
V_{\text {cell }}=\phi_{s, \text { cat }}-\phi_{s, \text { ano }}-I R_{c}=\phi_{s, \text { cat }}-I R_{c}
\end{gathered}
$$

Species conservation in solid phase.-Lithium transport inside active material particle is solved assuming spherical geometry with flux governed by Fick's law of diffusion.

$$
\begin{gathered}
\frac{\partial c_{s}}{\partial t}=\frac{1}{r^{2}} \frac{\partial}{\partial r}\left(D_{s} r^{2} \frac{\partial c_{s}}{\partial r}\right) \\
\mathrm{BC}: r=0: \frac{\partial c_{s}}{\partial r}=0 ; r=R_{\text {mean }}: \frac{\partial c_{s}}{\partial r}=-\frac{i}{D_{s} F}
\end{gathered}
$$

Species conservation in electrolyte phase.-Diffusion of lithium ions inside the electrolyte is also modeled using Fick's law with $D_{e}^{e f f}$ giving the effective diffusion rate of lithium ions in the electrolyte phase. Volumetric current production/consumption is given by $j$.

$$
\begin{gathered}
\varepsilon \frac{\partial c_{e}}{\partial t}=\nabla \cdot\left(D_{e}{ }^{e f f} \nabla c_{e}\right)+\frac{1-t^{+}}{F} j \\
j=a_{s} i \\
a_{s}=3 \varepsilon_{S} / R_{\text {mean }}
\end{gathered}
$$

Charge conservation in solid phase.-Electric potential in the solid phase $\phi_{s}$ can be determined using Ohm's law.

$$
\nabla \cdot\left(\sigma_{s}^{e f f} \nabla \phi_{s}\right)-j=0
$$

Charge conservation in electrolyte phase.-Charge motion in the electrolyte phase is driven by gradients in electrolyte potential $\phi_{e}$ and ionic concentration, $c_{e}$.

$$
\begin{gathered}
\nabla \cdot\left(\kappa^{e f f} \nabla \phi_{e}\right)+\nabla \cdot\left(\kappa_{D}{ }^{e f f} \nabla \ln c_{e}\right)+j=0 \\
V=\left(1-t^{+}\right)\left(1+\frac{d \ln f_{ \pm}}{d \ln c}\right) \\
\kappa_{D}^{e f f}=\frac{2 \Re T \kappa^{e f f}}{F}\left(t^{+}-1\right)\left(1+\frac{d \ln f_{ \pm}}{d \ln c_{e}}\right)
\end{gathered}
$$

The electrolyte properties and boundary conditions are listed in Tables IV and V respectively. Electrolyte properties were measured by Dees et al. ${ }^{40}$ Temperature evolution from the energy conservation equation is ignored since all the tests were performed using coin cells. Temperature rise is negligible in these small cells because the cells have a very low gravimetric energy density and are tested at very low power in an environment where the temperature is controlled.

The pseudo 2D model (P2D) converges to the single particle model $(\mathrm{SPM})$ at low current densities $(<1 \mathrm{C})$ due to negligible variations in 
Table IV. Electrolyte Properties.

Parameters $c:$ moles $/ \mathrm{cm}^{3}, T: \mathrm{K}$

Value

$\begin{array}{cc}D_{e}\left(\mathrm{~cm}^{2} / \mathrm{s}\right) & 0.00584 \mathrm{e}^{(-2870 / T)}(1000 c)^{2}-0.0339 \mathrm{e}^{(-2920 / T)}(1000 c)+0.129 \mathrm{e}^{(-3200 / T)} \\ \kappa(\mathrm{S} / \mathrm{cm}) & 34.5 e^{(-798 / T)}(1000 c)^{3}-485 e^{(-1080 / T)}(1000 c)^{2}+2440 \mathrm{e}^{(-1440 / T)}(1000 c) \\ \frac{\partial \ln f_{ \pm}}{\partial \ln c}(-) & -0.000267 \mathrm{e}^{(883 / T)}(1000 c)^{2}+0.00309 \mathrm{e}^{(653 / T)}(1000 c)+0.517 \mathrm{e}^{(-49.6 / T)} \\ t^{+}(-) & 0.540 \mathrm{e}^{(329 / T)}(1000 c)^{2}-0.00225 \mathrm{e}^{(1360 / T)}(1000 c)+0.341 \mathrm{e}^{(261 / T)}-1\end{array}$

Table V. Boundary Conditions.

Parameters

$\begin{array}{cc} & -\left(D_{e}^{e f f} \frac{\partial c_{e}}{\partial x}\right) \\ c_{e} & =\frac{1-t^{+}}{F} \frac{I}{S_{c s a}} \\ & -\left(\kappa^{e f f} \nabla \phi_{e}+\kappa_{D}^{e f f} \nabla \ln c_{e}\right) \\ \phi_{e} & =I / S_{c s a} \\ \phi_{s} & -\end{array}$

electrolyte phase concentration and potential. ${ }^{38}$ Consequently, the current density can be assumed to be uniform throughout the thickness of the electrode and transport in the thickness direction is ultimately neglected in the single particle model. Solid phase diffusion and charge transfer kinetics are the only phenomena of consequence in the single particle approximation, hence the solution algorithm is greatly simplified. Thus, performance modeling of LIBs using SPM can be accomplished by coupling the spherical diffusion equation with the Butler-Volmer equation. This significantly reduces the number of parameters for the simulations and hence SPM is an attractive option for modeling GITT datasets, which invariably have low current densities.

Figures 11 and 12 compare the results of the complete experimental GITT datasets during charge and discharge with the single particle model simulation results. Voltage mismatch between the experimental and predicted voltage is also plotted as a function of time. Robust match is obtained between the experimental and model results. Figure $11 \mathrm{~b}$ exhibits a closer look on the comparison for 10 charge current pulses. Here again, the model and experiment results show good agreement. The efficacy of our model is hence established. The parameters used in the models are surface roughness, $\psi$ and contact resistance $R_{c}$. The surface roughness value determines the active interfacial area which is then subsequently used to compute the actual diffusivity and intercalation rate constant via the correlations obtained from the experimental GITT dataset. The benefit of this method lies in the fact that a single parameter, the surface roughness, $\psi$, is being used to adjust three quantities: interfacial area, diffusivity and Li intercalation rate constant.

Finally, Figures 13 and 14 compare the experimental half-cell performance results with the single particle and pseudo 2D models. P2D model becomes necessary at current rates beyond $1 \mathrm{C}$ where deviation in the results predicted by the two models become apparent. The rate capability study done here ranges from $\mathrm{C} / 27$ to $1.5 \mathrm{C}$, hence it was considered best to use both models to check the validity of our formulation. Tortuosity used in the P2D model is calculated according to the Bruggeman relation.

$$
\tau=\varepsilon^{-0.5}
$$

The constant current discharge results show reasonable agreement for both the SPM and P2D models. Surface roughness $(\psi)$ value ranging from 1.17-1.25 provided a decent fit with the experimental datasets for the entire range of currents. Predominantly, the surface roughness magnitude affected the voltage profile at higher $\mathrm{C}$-rates. For low current $(<1 \mathrm{~mA})$, a wider range of surface roughness values $(1.10$ 1.30) were able to provide decent agreement with the experiments. It has to be noted that this surface roughness value is considerably smaller than the upper bound of surface roughness $\left(\psi_{\max }=1.841\right)$ computed according to Equation 26. This can be attributed to a few factors missing in our surface area estimation like particle-to-particle
Separator-cathode $x=L_{s}$

Cathode-CC $x=L_{s}+L_{c}$

$$
\begin{gathered}
\left(D_{e}^{e f f} \frac{\partial c_{e}}{\partial x}\right)_{x=L_{s}-\delta} \\
=\left(D_{e}^{e f f} \frac{\partial c_{e}}{\partial x}\right)_{x=L_{s}+\delta} \\
\left(\kappa^{e f f} \nabla \phi_{e}+\kappa_{D}^{e f f} \nabla \ln c_{e}\right)_{L_{s}-\delta} \\
=\left(\kappa^{e f f} \nabla \phi_{e}+\kappa_{D}^{e f f} \nabla \ln c_{e}\right)_{L_{s}+\delta} \\
\frac{\partial \phi_{s}}{\partial x}=0
\end{gathered}
$$$$
\begin{gathered}
\frac{\partial c_{e}}{\partial x}=0 \\
\frac{\partial \phi_{e}}{\partial x}=0 \\
\phi_{e}=0 \\
\sigma_{s}^{e f f} \frac{\partial \phi_{s}}{\partial x}=-\frac{I}{A}
\end{gathered}
$$

overlap and active material coverage with binder which can reduce the active area. Nevertheless, the obtained surface roughness value greater than 1 indicates that the overall active area in NCM523 particles is larger in magnitude than area computations done considering only spherical secondary particle dimension as the mean particle size. Thus, surface roughness enabled active area formulation is able to mimic the

(a)

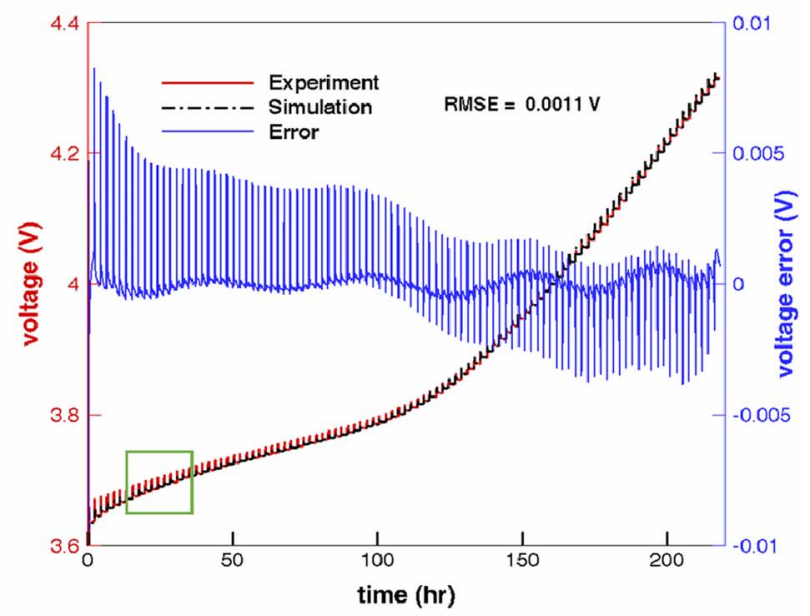

(b)

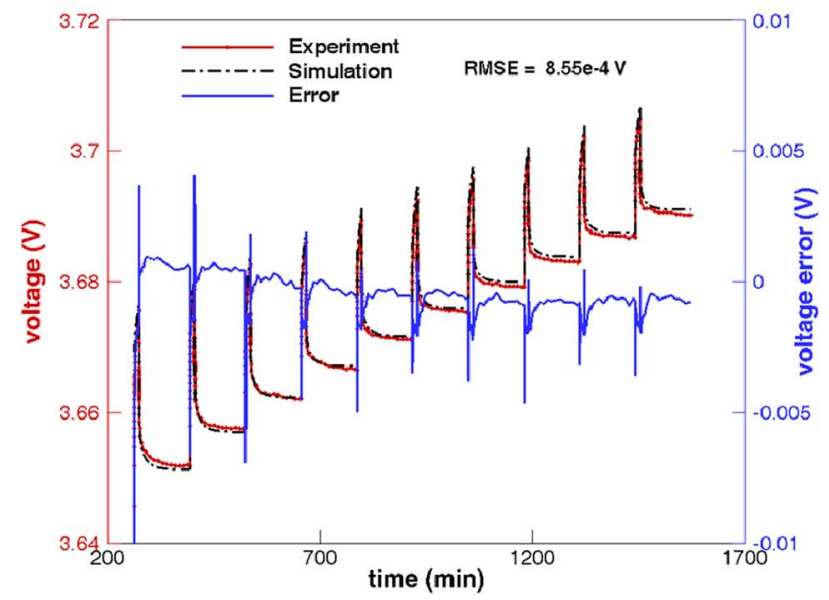

Figure 11. Comparison of experimental GITT results with simulated data using single particle model for (a) charge and (b) 10 charge pulses (inset). 


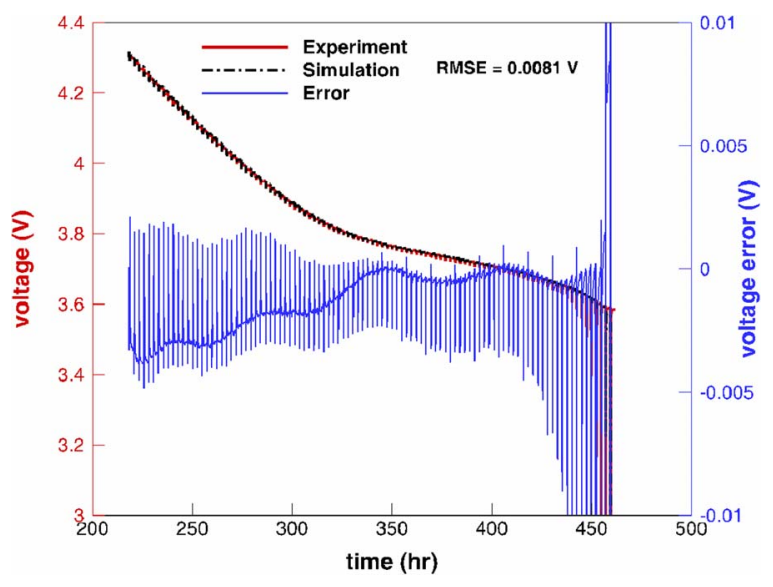

Figure 12. Comparison for discharge charge pulses of experimental GITT results with simulated data using single particle model. Larger error magnitude is seen as compared to charge because of wider variation near end of discharge.

(a)

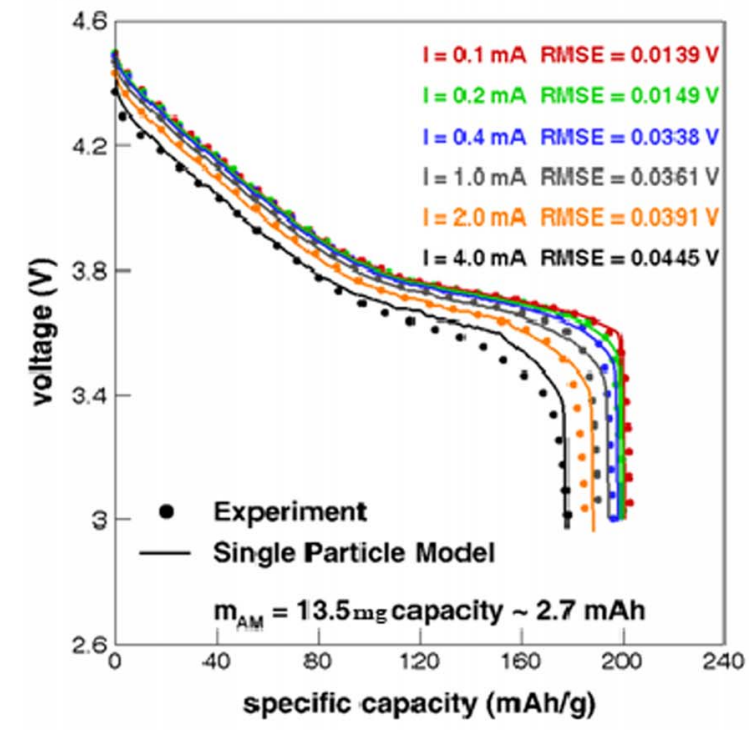

(b)

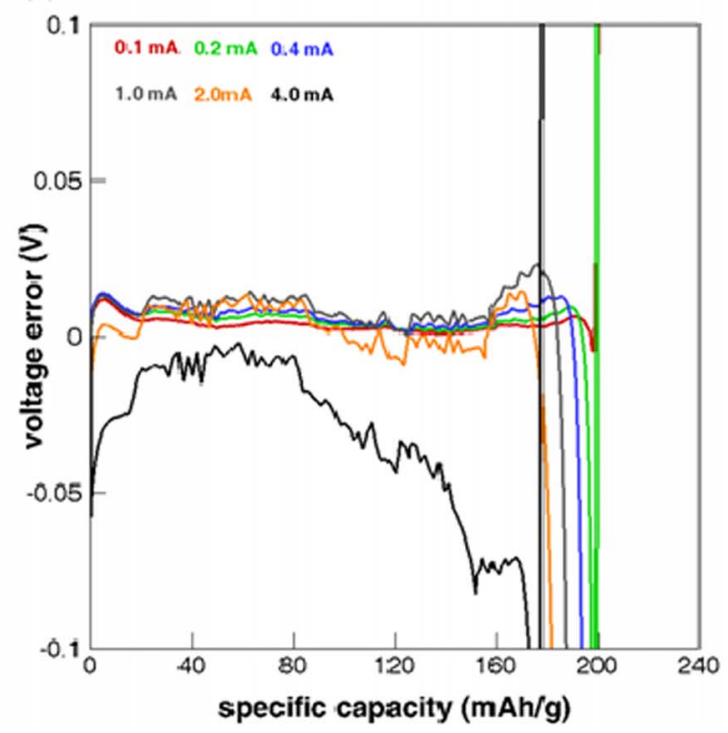

Figure 13. Comparison of experimental NCM523 half-cell results with macrohomogeneous single particle model (a) voltage vs specific capacity (b) voltage error. (a)

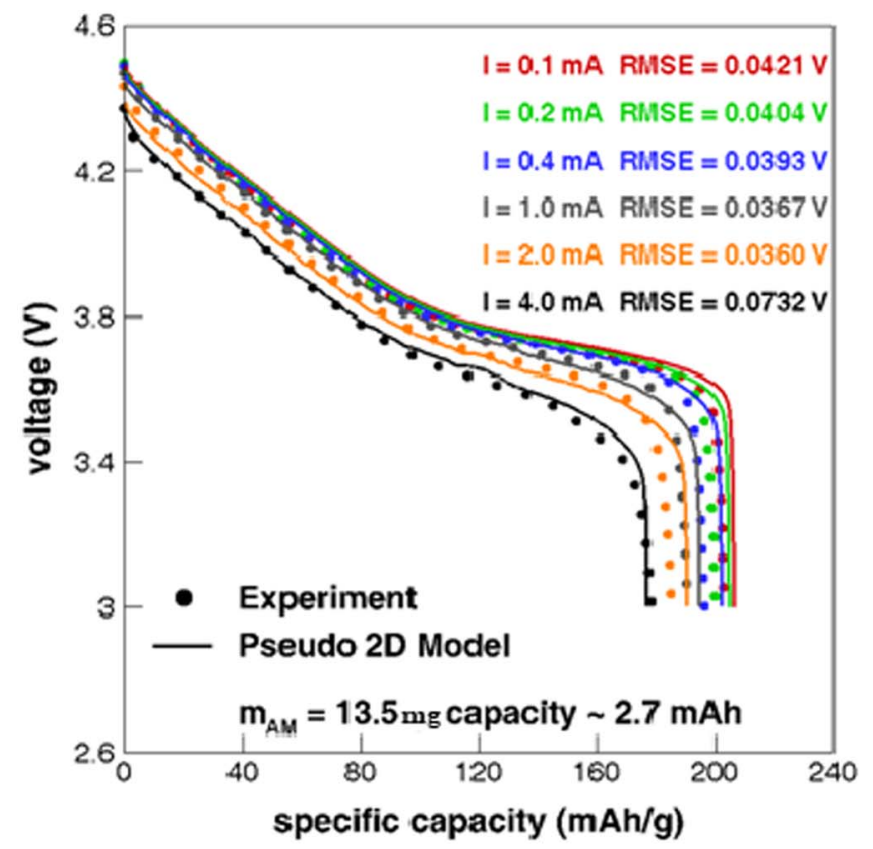

(b)

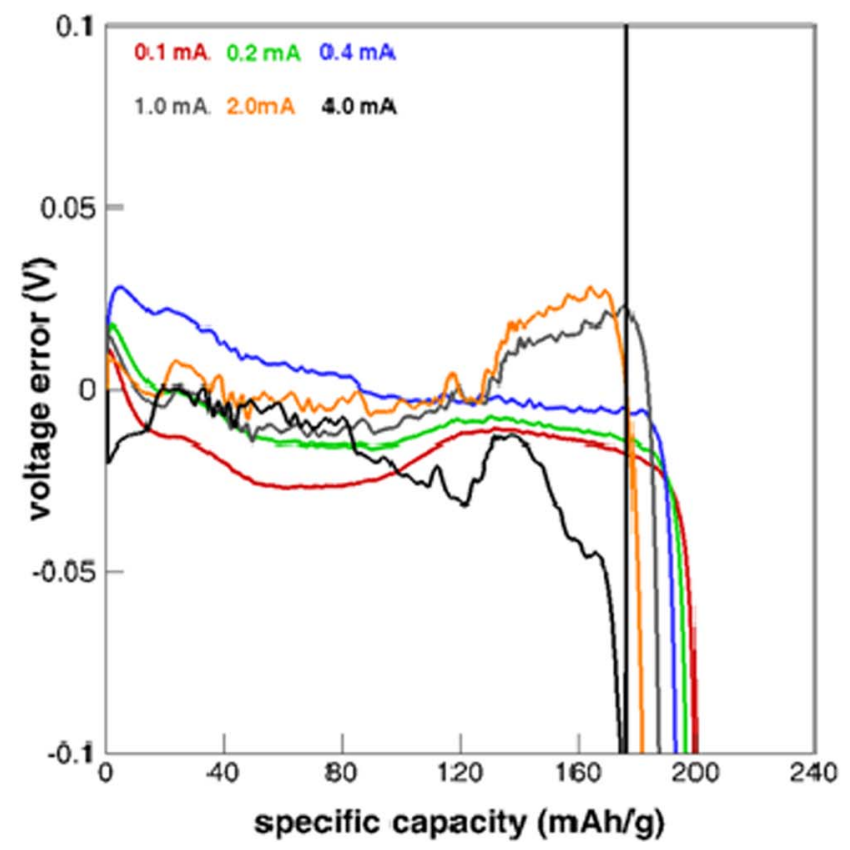

Figure 14. Comparison of experimental NCM523 half-cell results with macrohomogeneous pseudo 2D model (a) voltage vs specific capacity (b) voltage error.

physics of NCM523 particles to some extent. Further analysis with thicker electrodes and higher $\mathrm{C}$-rates will be important in gauging the effectiveness of this model formulation.

Figure 15 compares the open circuit potential as well as diffusivity obtained in this work with Yang et al. ${ }^{22}$ data. The open circuit potential shows a good match with the Yang et al. ${ }^{22}$ data. However, the diffusivity shows different magnitudes because we have estimated the active area $\left(=19.14 \mathrm{~cm}^{2}\right)$ based on our model fit with experimental GITT and rate performance data while Yang et al. ${ }^{22}$ used cell geometric area $\left(=0.785 \mathrm{~cm}^{2}\right)$ for its diffusivity calculations. 
(a)

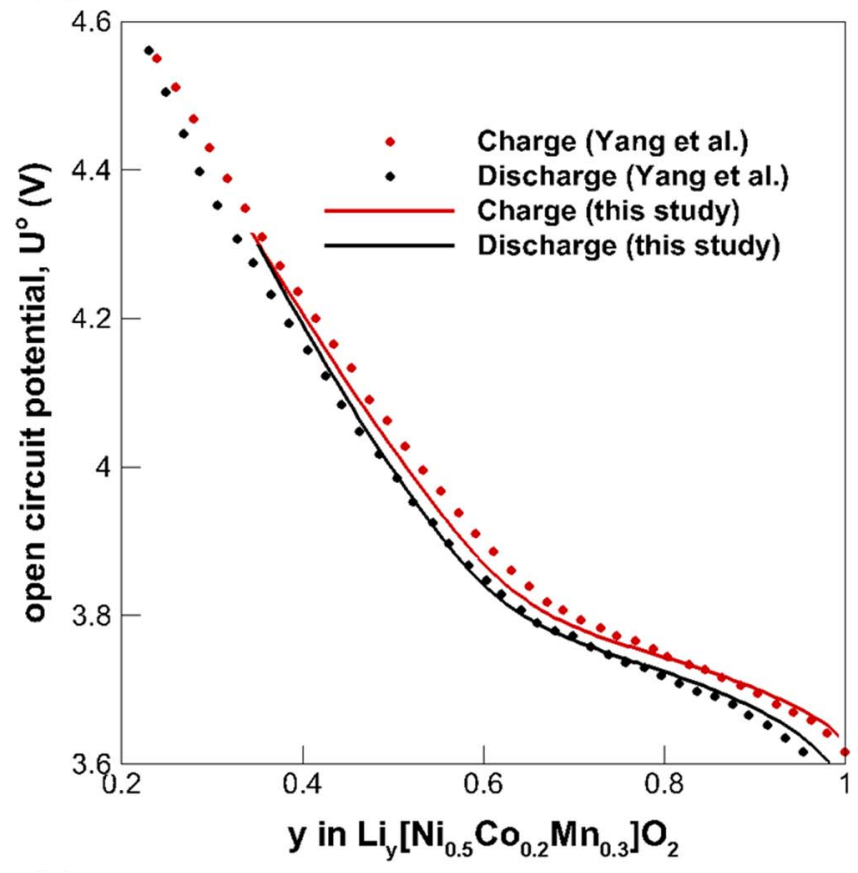

(b)

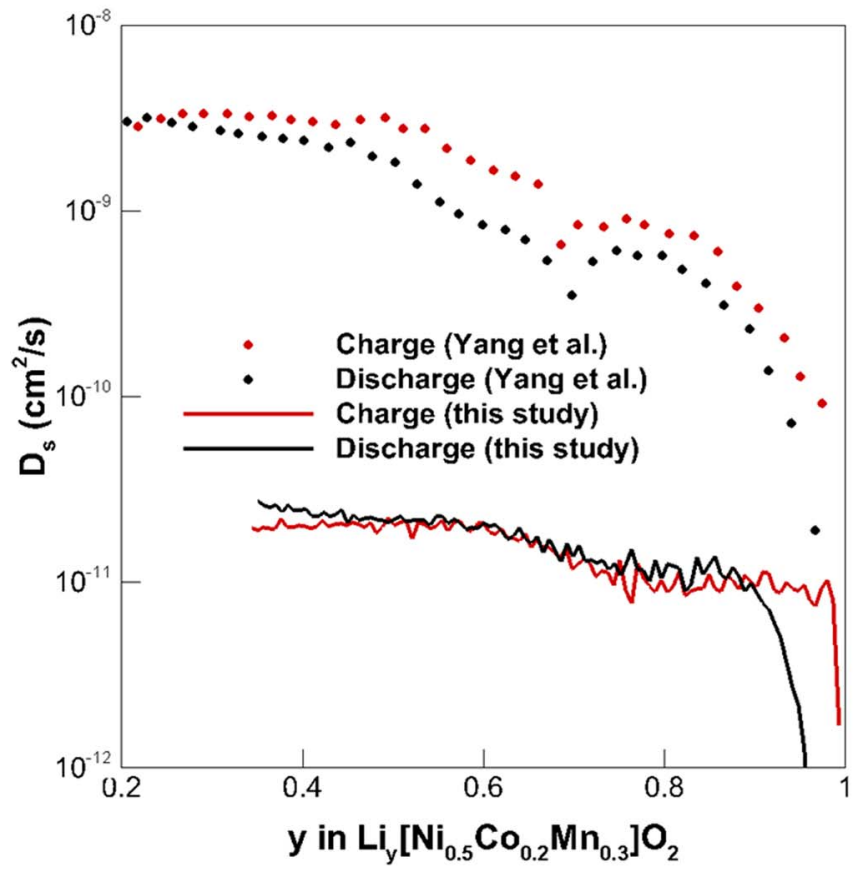

Figure 15. (a) Comparison of open circuit potential between Yang et al. ${ }^{22}$ and our dataset. Close match is obtained over the state of charge range investigated. (b) Comparison of diffusivity data between Yang et al. and our dataset. The difference is because Yang et al. ${ }^{22}$ used geometric area for its diffusivity computations.

A short note on the effect of particle size distribution on GITT calculations is given here. In our model, we have tried to incorporate the size effects by considering a mean value for the particle size with surface roughness in the area formulation. This model is able to mimic the experimental GITT and rate performance data reasonably well. However, a more accurate computation would be incorporating the complete particle size distribution in the diffusivity calculations. Presence of particle size distribution will lead to the particles expe-

riencing different current density at the particle surface depending on the size. Formulation of a GITT model incorporating particle size distribution is the way forward and will be investigated in a future work. Particle size distribution effects can be expected to have more significance than surface roughness effect.

\section{Conclusions}

We have developed a performance model that is successful in capturing the bimodal distribution of the NCM523 particles. The modified area computations help in ascertaining accurate kinetic and transport properties of the NCM523 electrode from the GITT dataset as well. This work highlights the need for coupling experimental dataset analysis with simulation model results so as to attain higher degrees of precision. Interfacial area has proved to be tough to estimate accurately, hence, a model utilizing area as an adjustable parameter to match electrochemical models to experimental data is essential to understanding the performance and rate limitations of NCM523 electrode materials.

\section{Acknowledgment}

This work was supported by the U.S. Department of Energy under Contract No. DE-AC36-08GO28308 with the National Renewable Energy Laboratory. Funding was provided by the U.S. DOE Office of Vehicle Technologies Energy Storage Program, program manager Brian Cunningham. The authors thank Dr. Ahmad Pesaran at NREL for his support. The authors also wish to acknowledge Bryant Polzin, Stephen Trask and Andy Jansen of the ANL CAMP facility for manufacturing the electrodes.

\section{List of Symbols} $c_{\text {cell }}$ $c_{e}$

$c_{s}$ $c_{s}^{\max }$ $\mathrm{d}$ $D_{s}$ $D_{e}$ $D_{e}{ }^{e f f}$ $\varepsilon$ $\varepsilon_{A M}$ $\eta$ F I $i$ $i_{o}$ j $k$
Specific surface area of electrode $\left(\mathrm{m}^{2} / \mathrm{m}^{3}\right)$ Capacity of NCM523 half cell $(\mathrm{mAh} / \mathrm{g})$ Electrolyte concentration $\left(\mathrm{mol} / \mathrm{m}^{3}\right)$ Concentration of $\mathrm{Li}$ in solid phase $\left(\mathrm{mol} / \mathrm{m}^{3}\right)$ Maximum Li solid phase concentration $\left(\mathrm{mol} / \mathrm{m}^{3}\right)$ Total derivative

Solid phase diffusivity $\left(\mathrm{m}^{2} / \mathrm{s}\right)$

Electrolyte diffusivity $\left(\mathrm{m}^{2} / \mathrm{s}\right)$

Effective electrolyte diffusivity $\left(\mathrm{m}^{2} / \mathrm{s}\right)$

Porosity (-)

Active material volume fraction (-)

Overpotential (V)

Faraday's constant $(96485.33 \mathrm{C} / \mathrm{mol})$

Current (A)

Current density $\left(\mathrm{A} / \mathrm{m}^{2}\right)$

Exchange current density $\left(\mathrm{A} / \mathrm{m}^{2}\right)$

Volumetric current density $\left(\mathrm{A} / \mathrm{m}^{3}\right)$

Reaction rate constant $\left(\mathrm{m}^{2.5} \mathrm{~mol}^{-0.5} \mathrm{~s}^{-1}\right)$

Separator thickness $(\mathrm{m})$

Cathode thickness (m)

Charge transfer resistance $\left(\Omega-\mathrm{cm}^{2}\right)$

Cell contact resistance $(\Omega)$

Particle radius (m)

Electrode active area $\left(\mathrm{m}^{2}\right)$

Cell cross sectional area $\left(\mathrm{m}^{2}\right)$

Time (s)

Transference number (-)

Temperature (K)

NCM523 Open circuit potential (V)

Voltage (Volts)/Volume $\left(\mathrm{m}^{3}\right)$

Molar volume $\left(\mathrm{m}^{3} / \mathrm{mol}\right)$

State of charge of lithium in NCM523 (-) 


\section{Greek}

$\alpha_{a}, \alpha_{c}$

$\kappa$

$\kappa_{D}$

$\phi_{s}$

$\phi_{e}$

$\psi$

$\mathfrak{R}$

$\sigma_{s}$

$\sigma_{s}^{e f f}$

$\theta_{s}$

$\partial$

$\nabla$

\begin{tabular}{ll} 
& \multicolumn{1}{r}{ Subscripts } \\
ano & Anode \\
agg & Agglomerate \\
cat & Cathode \\
e & Electrolyte \\
pr & Primary \\
proj & Projected \\
s & Solid/Sphere \\
sep & Separator \\
sc & Secondary \\
surf & Surface
\end{tabular}

\section{Superscripts}

eff $\max$ $(0.5,0.5)$

Surface roughness (-)

Partial derivative

Gradient

\section{Subscripts}

Agglomerate

Cathode

Projected

Separator

Secondary

Effective property

Maximum
Anodic and cathodic charge transfer coefficients

Ionic conductivity $(\mathrm{S} / \mathrm{m})$

Diffusional conductivity $(\mathrm{A} / \mathrm{m})$

Solid phase potential (V)

Electrolyte phase potential (V)

Universal gas constant $(8.314 \mathrm{~J} / \mathrm{mol}-\mathrm{K})$

Electronic conductivity $(\mathrm{S} / \mathrm{m})$

Effective electronic conductivity $(\mathrm{S} / \mathrm{m})$

Surface stoichiometry of spherical particle (-)

\section{References}

1. M. Armand and J.-M. Tarascon, Nature, 451, 652 (2008).

2. L. Lu, X. Han, J. Li, J. Hua, and M. Ouyang, Journal of Power Sources, 226, 272 (2013).

3. M. Yoshio, H. Wang, and K. Fukuda, Angewandte Chemie, 115, 4335 (2003).

4. J.-i. Yamaki, H. Takatsuji, T. Kawamura, and M. Egashira, Solid State Ionics, 148, 241 (2002).

5. K. Ozawa, Solid State Ionics, 69, 212 (1994).

6. D. K. Kim, P. Muralidharan, H.-W. Lee, R. Ruffo, Y. Yang, C. K. Chan, H. Peng, R. A. Huggins, and Y. Cui, Nano Letters, 8, 3948 (2008).

7. V. Srinivasan and J. Newman, Journal of the Electrochemical Society, 151, A1517 (2004).

8. R. Kostecki and F. McLarnon, Electrochemical and Solid-State Letters, 5, A164 (2002).

9. S. Albrecht, J. Kümpers, M. Kruft, S. Malcus, C. Vogler, M. Wahl, and M. Wohlfahrt-Mehrens, Journal of Power Sources, 119, 178 (2003).
10. L. Wang, Z. Tang, L. Ma, and X. Zhang, Electrochemistry Communications, 13, 1233 (2011).

11. H.-J. Noh, S. Youn, C. S. Yoon, and Y.-K. Sun, Journal of Power Sources, 233, 121 (2013).

12. S.-L. Wu, W. Zhang, X. Song, A. K. Shukla, G. Liu, V. Battaglia, and V. Srinivasan, Journal of The Electrochemical Society, 159, A438 (2012).

13. J. Chen, Materials, 6, 156 (2013).

14. A. Yaqub, Y.-J. Lee, M. J. Hwang, S. A. Pervez, U. Farooq, J.-H. Choi, D. Kim, H.-Y. Choi, S.-B. Cho, and C.-H. Doh, Journal of Materials Science, 49, 7707 (2014).

15. Y. Leng, S. Ge, D. Marple, X.-G. Yang, C. Bauer, P. Lamp, and C.-Y. Wang, Journal of The Electrochemical Society, 164, A1037 (2017).

16. M. Dixit, M. Kosa, O. S. Lavi, B. Markovsky, D. Aurbach, and D. T. Major, Physical Chemistry Chemical Physics, 18, 6799 (2016).

17. Z. Wu, X. Han, J. Zheng, Y. Wei, R. Qiao, F. Shen, J. Dai, L. Hu, K. Xu, and Y. Lin, Nano letters, 14, 4700 (2014).

18. C. Liang, L. Liu, Z. Jia, C. Dai, and Y. Xiong, Electrochimica Acta, 186, 413 (2015).

19. J. C. Garcia, J. G. Bareno, J. Yan, G. Chen, A. Hauser, J. R. Croy, and H. Iddir, The Journal of Physical Chemistry C, 121, 8290 (2017).

20. S. K. Jung, H. Gwon, J. Hong, K. Y. Park, D. H. Seo, H. Kim, J. Hyun, W. Yang, and K. Kang, Advanced Energy Materials, 4 (2014).

21. J. Kasnatscheew, U. Rodehorst, B. Streipert, S. Wiemers-Meyer, R. Jakelski, R. Wagner, I. C. Laskovic, and M. Winter, Journal of The Electrochemical Society, 163, A2943 (2016).

22. S. Yang, X. Wang, X. Yang, Y. Bai, Z. Liu, H. Shu, and Q. Wei, Electrochimica Acta, 66, 88 (2012).

23. Y. Bai, X. Wang, X. Zhang, H. Shu, X. Yang, B. Hu, Q. Wei, H. Wu, and Y. Song, Electrochimica Acta, 109, 355 (2013).

24. J.-Z. Kong, C. Ren, G.-A. Tai, X. Zhang, A.-D. Li, D. Wu, H. Li, and F. Zhou, Journal of Power Sources, 266, 433 (2014).

25. L. Xia, K. Qiu, Y. Gao, X. He, and F. Zhou, Journal of Materials Science, 50, 2914 (2015).

26. R. Amin and Y.-M. Chiang, Journal of The Electrochemical Society, 163, A1512 (2016).

27. J. A. Gilbert, J. Bareño, T. Spila, S. E. Trask, D. J. Miller, B. J. Polzin, A. N. Jansen, and D. P. Abraham, Journal of The Electrochemical Society, 164, A6054 (2017).

28. P. Hovington, V. Timoshevskii, S. Burgess, H. Demers, P. Statham, R. Gauvin, and K. Zaghib, Scanning, 38, 571 (2016).

29. W. Weppner and R. A. Huggins, Journal of The Electrochemical Society, 124, 1569 (1977).

30. D. W. Dees, S. Kawauchi, D. P. Abraham, and J. Prakash, Journal of Power Sources, 189, 263 (2009).

31. Y. Zhu and C. Wang, The Journal of Physical Chemistry C, 114, 2830 (2010).

32. K. Dokko, M. Mohamedi, Y. Fujita, T. Itoh, M. Nishizawa, M. Umeda, and I. Uchida, Journal of the Electrochemical Society, 148, A422 (2001).

33. K. Shaju, G. S. Rao, and B. Chowdari, Electrochimica Acta, 49, 1565 (2004).

34. K. Shaju, G. S. Rao, and B. Chowdari, Journal of The Electrochemical Society, 151, A1324 (2004)

35. C.-F. Chen, A. Verma, and P. P. Mukherjee, Journal of The Electrochemical Society, 164, E3146 (2017)

36. S. Brunauer, P. H. Emmett, and E. Teller, Journal of the American chemical society, 60, 309 (1938).

37. M. Guo, G. Sikha, and R. E. White, Journal of The Electrochemical Society, 158, A122 (2011).

38. S. Santhanagopalan, Q. Guo, P. Ramadass, and R. E. White, Journal of Power Sources, 156, 620 (2006).

39. M. Doyle, T. F. Fuller, and J. Newman, Journal of the Electrochemical Society, 140, 1526 (1993)

40. D. Dees, E. Gunen, D. Abraham, A. Jansen, and J. Prakash, Journal of the Electrochemical Society, 155, A603 (2008). 